\title{
Conversion of 4-Oxoproline Esters to 4-Substituted Pyrrole-2-carboxylic Acid Esters
}

\author{
Yasushi Arakawa,* Naomi Yagi, Yukimi Arakawa, Ken-ichi Tanaka, and Shigeyuki Yoshifuji \\ Faculty of Pharmaceutical Sciences, Hokuriku University; Kanagawa-machi Ho-3, Kanazawa 920-1181, Japan. \\ Received September 29, 2008; accepted November 28, 2008; published online December 1, 2008
}

The Grignard, Wittig, Tebbe, Horner-Emmons, and Reformatsky reactions of the 4-oxoproline esters gave the corresponding 4-alylated or 4-alkylidenated products, respectively. The products were properly treated with bases to cause aromatization, giving 4-substituted pyrrole-2-carboxylic acid esters such as methyl 4-methylpyrrole-2-carboxylate, which is a trail pheromone of Atta texana.

Key words 4-oxoproline ester; Grignard reaction; Horner-Emmons reaction; Reformatsky reaction; aromatization; methyl 4methylpyrrole-2-carboxylate

During our study of the synthesis of several compounds using 4-hydroxyproline (1) as a starting material, ${ }^{1)}$ when a $p$ toluenesulfonyl (Ts, tosyl) group was introduced to the nitrogen atom of 1 by the Schotten-Baumann method employing $\mathrm{TsCl}$ and aqueous $\mathrm{NaOH}$ according to Portoghese's method ${ }^{2)}$ in order to obtain compound $\mathbf{2}$, thereby prolonging the reaction, we encountered a side reaction that caused the elimination of sulfinic acid and aromatization, giving pyrrole-2-carboxylic acid (3) (Chart 1). We therefore considered that the aromatization could be utilized for the synthesis of pyrrole2-carboxylic acid derivatives such as methyl 4-methylpyrrole-2-carboxylate (4), which is a trail pheromone of Atta texana. ${ }^{3-6)}$

Although aromatizations of the $N$-tosylproline derivatives giving pyrrole-2-carboxylic acid derivatives, ${ }^{7-9}$ ) as well as other syntheses of the pyrrole-2-carboxylic acid derivatives, ${ }^{10-12)}$ have been reported by many workers, synthesis of the pyrrole-2-carboxylic acid derivatives using 4-oxoproline derivatives has not been reported. Therefore, we performed alkylation and alkylidenaion of $N$-tosyl-4-oxoproline ester, followed by aromatization of the products to give 4-substituted proline-2-carboxylic acid esters 7 (Chart 2). This method made it possible to introduce not only a simple alkyl group but also an alkyl possessing a functional group into 4position of the pyrrole-2-carboxylic acid derivatives.

Thus we report here the conversion of 4-oxoproline esters 5 and 6 to the 4-substituted proline-2-carboxylic acid esters 7.

Base Treatments of $\boldsymbol{N}$-Tosyl-4-oxoproline Esters We chose the methyl and/or tert-butyl esters of $N$-tosyl-4-oxo-

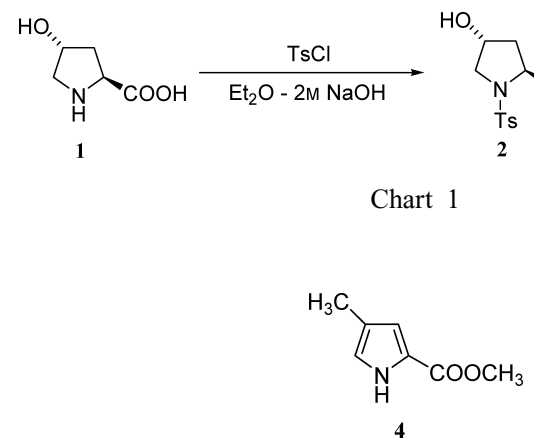

Fig. 1. Trail Pheromone of Atta texana

* To whom correspondence should be addressed. e-mail: ya-arakawa@hokuriku-u.ac.jp proline $\mathbf{5}^{13)}$ and $\mathbf{6}^{14)}$ as substrates for the ketone reactions, and synthesized them from 1 according to the previously reported methods. ${ }^{2}$ Next, we attempted the base treatments of 5 and 6 using appropriate bases to cause enolization and aromatization. As a result, 4-hydroxypyrrole-2-carboxylic acid esters 8 and 9 were successfully obtained in $76 \%$ and $60 \%$ yields, respectively. Only the benzyl ester of 4-hydroxypyrrole-2-carboxylic acid ${ }^{15)}$ has been reported previously; 8 and $\mathbf{9}$ are new compounds (Chart 3 ).

The Grignard Reactions of $N$-Tosyl-4-oxoproline Ester and Conversion into the Pyrrole Derivatives Grignard reactions were attempted with the tert-butyl ester of $N$-tosyl-4oxoproline 6, in order to prevent the reaction at the ester moiety. When the Grignard reagent was employed without addition of other reagent, the by-products were remarkably formed and yields of the desired products were much lower (mixture of epimers $<70 \%$ ). In order to prevent the aldol reaction and the aromatization of $\mathbf{6}$, which seemed to be the side reactions, additions of $\mathrm{CeCl}_{3}{ }^{16,17)}$ into the reaction systems were examined. Consequently, the numbers of the com-
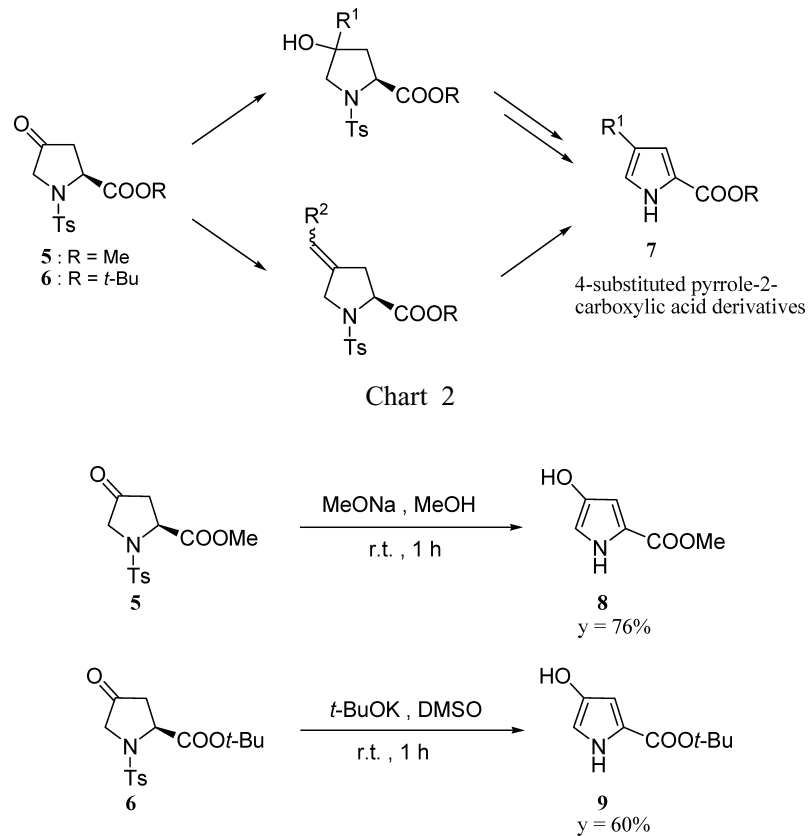

Chart 3 


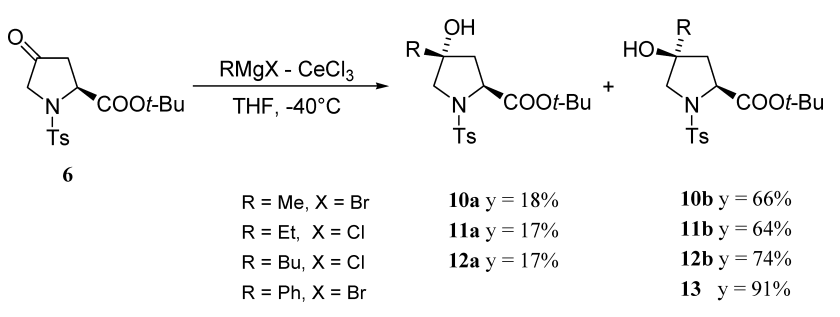

Chart 4. The Grignard Reactions of Compound 6

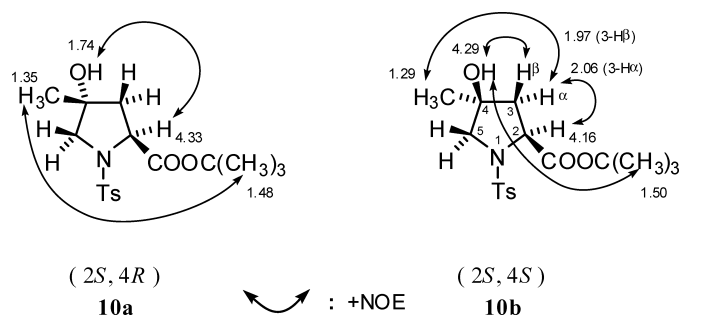

Fig. 2. Selected Decisive NOE Relationships of Compounds 10a and 10b

ponents of the reaction mixtures decreased in the TLC analyses and the yields of the desired products improved (Chart 4).

The obtained products were the epimers in each alkylation, and the ratio of the epimers seemed to change by the size of the alkyl (or phenyl) groups introduced to the 4-position. When a phenyl group was introduced, a single epimer, $(2 S, 4 R)$-form 13 was generated. Although, by the IUPAC nomenclature, the absolute configuration of $\mathbf{1 3}$ is different from those of $\mathbf{1 0 b}-\mathbf{1 2 b}$, which were $(2 S, 4 S)$-forms, the structure of 13 should be similar to those of $\mathbf{1 0 b}-\mathbf{1 2 b}$. The stereochemistries of the epimers were determined by differential nuclear Overhauser effect (NOE) experiments of ${ }^{1} \mathrm{H}-$ NMR (Fig. 2). As for 10a, + NOEs were observed between a signal at $\delta 1.74$ assigned to the hydroxy proton and a signal at $\delta 4.33$ assigned to $2-\mathrm{H}$, and between a signal at $\delta 1.35$ assigned to the 4-methyl protons and a signal at $\delta 1.48$ assigned to methyl protons of the tert-butyl group. Therefore, $\mathbf{1 0 a}$ was proved to have a $(2 S, 4 R)$-configuration. As for $\mathbf{1 0 b}$, + NOEs were observed between a signal at $\delta 4.29$ assigned to the hydroxy proton and signals at $\delta 1.97$ assigned to $3-\mathrm{H}_{\beta}$ and $\delta 1.50$ assigned to methyl protons of the tert-butyl group. + NOEs were also observed between a signal at $\delta$ 2.06 assigned to $3-\mathrm{H}_{\alpha}$ and signals at $\delta 4.16$ assigned to $2-\mathrm{H}$ and $\delta 1.29$ assigned to 4-methyl protons. Therefore, $\mathbf{1 0 b}$ was proved to have a $(2 S, 4 S)$-configuration. In the case of 1113, +NOEs similar to those for compound $\mathbf{1 0}$ were observed, and the configurations of these compounds were confirmed.

Further, differences between the characteristics of the epimers 10a-12a and 10b-12b were observed in TLC (silica gel) analysis and IR spectra due to the probable intramolecular hydrogen bondings between a hydroxy group and an ester carbonyl group of $\mathbf{1 0 b}-\mathbf{1 2 b}$. The $R f$ values of $\mathbf{1 0 b}$ 12b were higher than those of the corresponding epimers 10a-12a in TLC analysis, because the intermolecular hydrogen bondings between the hydroxy groups of $\mathbf{1 0 b}-\mathbf{1 2 b}$ and silica gel seem to have been prevented. Concerning the characteristic absorption bands of carbonyl groups in the IR spectra, those of $\mathbf{1 0 b}-\mathbf{1 2 b}\left(1707,1720,1718 \mathrm{~cm}^{-1}\right)$ were located at lower wavenumbers than those of 10a-12a (1728, $\left.1751,1730 \mathrm{~cm}^{-1}\right)$.

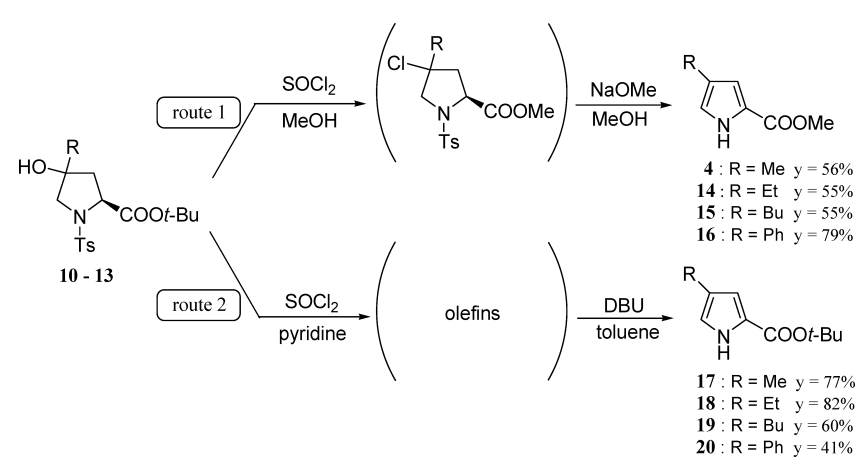

Chart 5

Next, aromatizations of the compounds 10-13 were attempted. In order to obtain methyl esters of 4-substituted pyrrole-2-carboxylic acids, $\mathbf{1 0 b}-\mathbf{1 2 b}$ and $\mathbf{1 3}$ were treated with $\mathrm{SOCl}_{2}-\mathrm{MeOH}$, which should cause chlorination and transesterification, then treated with $\mathrm{NaOMe}$ in $\mathrm{MeOH}$, affording compounds $4, \mathbf{1 4}, \mathbf{1 5}$, and 16 in $56 \%, 55 \%, 55 \%$, and $79 \%$ yields, respectively (Chart 5 , route 1). Compounds 10a-12a were also treated similarly to $\mathbf{1 0 b}-\mathbf{1 2 b}$, and similar results were obtained.

Meanwhile, to obtain tert-butyl esters of 4-substituted pyrrole-2-carboxylic acids, $\mathbf{1 0 b}-\mathbf{1 2 b}$ and $\mathbf{1 3}$ were treated with $\mathrm{SOCl}_{2}$-pyridine, which should cause chlorination and elimination, then treated with 1,8-diazabicyclo[5.4.0]undec-7-ene (DBU) in refluxing toluene, affording compounds 17, 18, 19, and 20 in $77 \%, 82 \%, 60 \%$, and $41 \%$ yields, respectively (Chart 5 , route 2 ).

Incidentally, the aromatizations are characteristic of $N$-tosylproline derivatives, and normally $N$-protected proline derivatives (urethanes and amides) do not undergo aromatization under the basic conditions. For example, treatment of $\mathrm{N}$ tert-butoxycarbonyl-4-iodo-L-proline methyl ester with DBU provided 3,4- and 4,5-dehydroproline derivatives without aromatization. ${ }^{18)}$

The Wittig, the Tebbe, and the Horner-Emmons Reactions of $N$-Tosyl-4-oxoproline Esters and Conversion into the Pyrrole Derivatives We examined the Wittig reaction of 5 with methylenetriphenylphosphorane, which was generated by treatment of methyltriphenylphosphonium bromide with phenyllithium in $\mathrm{Et}_{2} \mathrm{O},{ }^{19)}$ at room temperature and the expected 4-methyleneproline derivative $\mathbf{2 1}$ was obtained in only $10 \%$ yield. A similar reaction was examined by Cheng et al., and they reported that the low yield of the reaction was due to the propensity of the sulfonamide moiety to decompose under basic conditions. ${ }^{20)}$

We next examined the Tebbe reaction ${ }^{21)}$ of $\mathbf{6}$, and the desired product 22 was obtained in 16\% yield together with the dimethylated product $\mathbf{2 3}$ in $22 \%$ yield (Chart 6).

Meanwhile, the Horner-Emmons reactions ${ }^{22)}$ of 5 and $\mathbf{6}$ gave satisfactory results, as shown in Table 1 . However, the expected products underwent rearrangement at olefin or aromatization under the basic reaction conditions to give the secondary products, depending on the alkyl groups of esters 5 and $\mathbf{6}$, temperatures, and reagents. For example, the Horner-Emmons reaction of $\mathbf{6}$ and triethyl phosphonoacetate at room temperature for $1 \mathrm{~h}$ (entry 3 ) gave exo-olefins 25a (4E) and 25b (4Z), endo-olefins 25c (3-ene) and 25d (4-ene), and pyrrole 29 , in $17 \%, 14 \%, 33 \%, 9 \%$, and $23 \%$ yields. A 


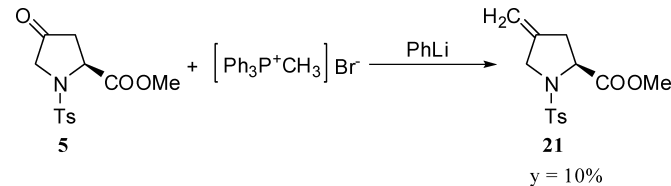

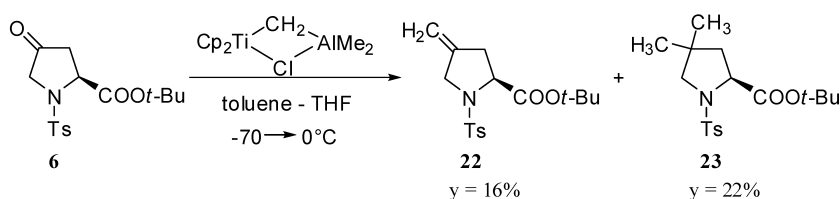

Chart 6
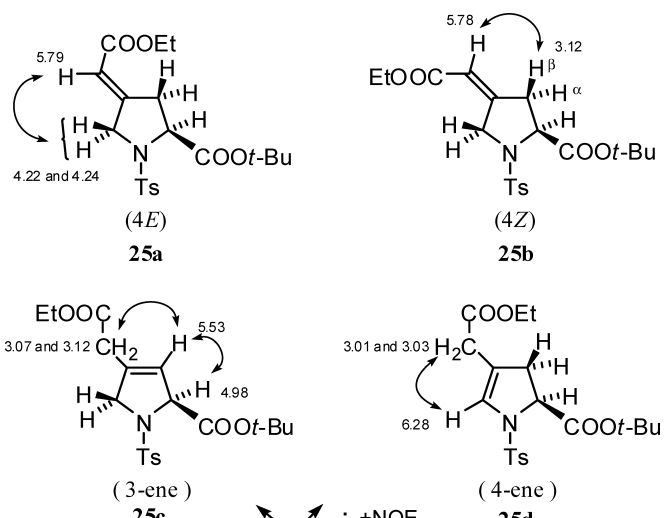

Fig. 3. Selected Decisive NOE Relationships of Compounds 25a-d

Table 1. The Horner-Emmons Reactions of Compounds 5 and $\mathbf{6}$

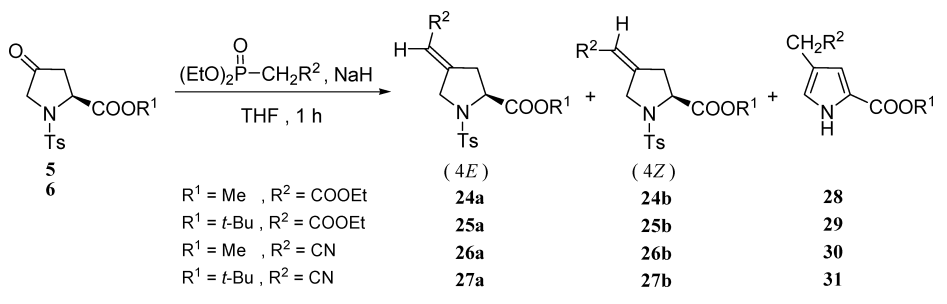

\begin{tabular}{|c|c|c|c|c|c|c|c|c|c|}
\hline \multirow{3}{*}{$\begin{array}{c}\text { Entry } \\
1\end{array}$} & \multirow{2}{*}{\multicolumn{2}{|c|}{$\begin{array}{c}\text { Substrate } \\
\mathrm{R}^{1}\end{array}$}} & \multirow{3}{*}{$\begin{array}{c}\mathrm{R}^{2} \\
\mathrm{COOEt}\end{array}$} & \multirow{4}{*}{$\begin{array}{c}\text { Temp. } \\
\text { r.t. } \\
-40^{\circ} \mathrm{C}\end{array}$} & \multicolumn{5}{|c|}{ Yields (\%) } \\
\hline & & & & & \multirow{2}{*}{$\frac{\text { Olefin }}{24}$} & \multirow{2}{*}{$\frac{\mathbf{a}(4 E)}{2}$} & \multirow{2}{*}{$\frac{\mathbf{b}(4 Z)}{-}$} & \multicolumn{2}{|c|}{ Pyrrole } \\
\hline & 5 & $\mathrm{Me}$ & & & & & & 28 & 86 \\
\hline 2 & & & & & & 48 & 44 & & - \\
\hline 3 & 6 & $t-\mathrm{Bu}$ & & r.t. & 25 & 17 & $14^{a)}$ & 29 & 23 \\
\hline 4 & & & & $0^{\circ} \mathrm{C}$ & & 37 & $30^{b)}$ & & - \\
\hline 5 & 5 & $\mathrm{Me}$ & $\mathrm{CN}$ & $0^{\circ} \mathrm{C}$ & 26 & - & 4 & 30 & 56 \\
\hline 6 & & & & $-40^{\circ} \mathrm{C}$ & & 37 & 33 & & 3 \\
\hline 7 & 6 & $t-\mathrm{Bu}$ & & $15^{\circ} \mathrm{C}$ & 27 & - & - & 31 & 98 \\
\hline 8 & & & & $0^{\circ} \mathrm{C}$ & & 48 & 36 & & 5 \\
\hline
\end{tabular}

a) The isomers and were obtained. b) $\mathbf{2 5 c} 30 \%, \mathbf{2 5 d} 2 \%$. r.t.: room temperature.

similar reaction at $0^{\circ} \mathrm{C}$ for $1 \mathrm{~h}$ (entry 4) gave 25a, 25b, 25c, and $25 \mathbf{d}$ in $37 \%, 30 \%, 30 \%$, and $2 \%$ yields without formation of 29. In the case of the reactions using diethyl cyanomethylphosphonate (entry 5-8), aromatization occurred more easily.

The structures of $\mathbf{2 4} \mathbf{- 2 7}$ were determined by differential nuclear Overhauser effect (NOE) experiments of ${ }^{1} \mathrm{H}-\mathrm{NMR}$ (Fig. 3). As for 25a, +NOEs were observed between a signal at $\delta 5.79$ assigned to the olefinic proton and signals at $\delta 4.22$ and $\delta 4.24$ assigned to 5 -Hs. For $25 \mathbf{b}$, + NOEs were observed between a signal at $\delta 5.78$ assigned to the olefinic proton and a signal at $\delta 3.12$ assigned to $3-\mathrm{H}_{\beta}$. For $25 \mathbf{c}$, + NOEs were observed between a signal at $\delta 5.53$ assigned to the olefinic 3-proton and signals at $\delta 3.07$ and $\delta 3.12$ assigned to methylene protons of the side chain, and $\delta 4.98$ assigned to 2-H. For 25d, +NOEs were observed between a signal at $\delta 6.28$ assigned to the olefinic 5-proton and signals at $\delta 3.01$ and $\delta 3.03$ assigned to methylene protons of the side chain, and $\delta 7.70$ assigned to aromatic protons. In the case of 24, 26, and 27, discriminations of the ( $4 E)$-form from the (4Z)-form were done similarly (Fig. 3).

Aromatizations of the olefinic compounds $\mathbf{2 1}$ and $\mathbf{2 2}$ were then examined (Table 2). In these cases, similarly to the aromatization process of 4-alkylated proline derivatives $\mathbf{1 0}$ 13, treatments of $\mathbf{2 1}$ and $\mathbf{2 2}$ with DBU in refluxing toluene for $12 \mathrm{~h}$ (entry 1 and 3 ) afforded pyrrole derivatives $\mathbf{4}$ and $\mathbf{1 7}$ in $97 \%$ and $99 \%$ yields, respectively. Without heating, the aromatization reaction hardly proceeded. Compounds $\mathbf{2 1}$ and 22 were also treated with potassium tert-butoxide in tetrahydrofuran (THF) at room temperature for $1 \mathrm{~h}$ (entry 2 and 4), giving 4 and 17 in $77 \%$ and $85 \%$ yields, respectively.

On the other hand, compounds $\mathbf{2 4 - 2 7}$ seemed to be more reactive (Table 3 ). Even at room temperature, treatments of 24a and 26a with DBU in toluene (using concentrations similar to that of 21) for $1 \mathrm{~h}$ (entry 1 and 3 ) gave the corresponding pyrrole derivatives $\mathbf{2 8}$ and $\mathbf{3 0}$ in $97 \%$ and $99 \%$ yields, 
Table 2. Aromatization of Compounds $\mathbf{2 1}$ and $\mathbf{2 2}$

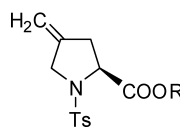

\begin{tabular}{ccclcc}
\hline \hline Entry & Substrate & $\mathrm{R}$ & \multicolumn{1}{c}{$\begin{array}{c}\text { Reaction } \\
\text { conditions }\end{array}$} & Product & $\begin{array}{c}\text { Yield } \\
(\%)\end{array}$ \\
\hline 1 & $\mathbf{2 1}$ & $\mathrm{Me}$ & $\begin{array}{l}\text { DBU, toluene, reflux, 12 h } \\
t \text {-BuOK, THF, r.t., 1 h }\end{array}$ & $\mathbf{4}$ & 97 \\
2 & \multirow{2}{*}{$t$-Bu } & $\begin{array}{l}\text { DBU, toluene, reflux, 12 h } \\
3\end{array}$ & $\mathbf{1 7}$ & 77 \\
4 & & & $t$-BuOK, THF, r.t., 1 h & & 89 \\
\hline
\end{tabular}

Table 3. Aromatization of Compounds 24-27

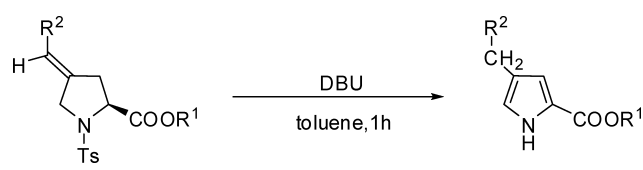

\begin{tabular}{ccccccc}
\hline \hline Entry & Substrate & $\mathrm{R}^{1}$ & $\mathrm{R}^{2}$ & Temp. & Product & $\begin{array}{c}\text { Yield } \\
(\%)\end{array}$ \\
\hline 1 & $\mathbf{2 4 a}$ & $\mathrm{Me}$ & COOEt & r.t. & $\mathbf{2 8}$ & 97 \\
2 & $\mathbf{2 5 a}$ & $t$-Bu & COOEt & Reflux & $\mathbf{2 9}$ & 99 \\
3 & $\mathbf{2 6 a}$ & $\mathrm{Me}$ & $\mathrm{CN}$ & r.t. & $\mathbf{3 0}$ & 99 \\
4 & $\mathbf{2 7 a}$ & $t$-Bu & $\mathrm{CN}$ & Reflux & $\mathbf{3 1}$ & 99
\end{tabular}

Table 4. The Reformatsky Reactions of Compound 6

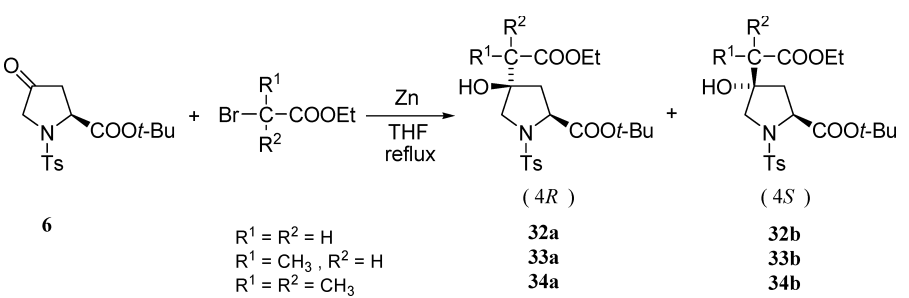

\begin{tabular}{|c|c|c|c|c|c|c|c|}
\hline \multirow{2}{*}{ Entry } & \multirow{2}{*}{$\mathrm{R}^{1}$} & \multirow{2}{*}{$\mathrm{R}^{2}$} & \multicolumn{2}{|c|}{ Reaction conditions } & & \multicolumn{2}{|c|}{ Yields (\%) } \\
\hline & & & Reagents & Time (h) & & $\mathbf{a}(4 R)$ & $\mathbf{b}(4 S)$ \\
\hline 1 & $\mathrm{H}$ & $\mathrm{H}$ & $\mathrm{Zn},\left(\mathrm{CH}_{3} \mathrm{O}\right)_{3} \mathrm{~B}$ & 1 & & \multicolumn{2}{|c|}{ Complicated mixture } \\
\hline 2 & & & $\mathrm{Zn}-\mathrm{Cu}$ & 1 & 32 & 45 & 10 \\
\hline 3 & $\mathrm{CH}_{3}$ & $\mathrm{H}$ & $\mathrm{Zn},\left(\mathrm{CH}_{3} \mathrm{O}\right)_{3} \mathrm{~B}$ & 1.5 & 33 & 55 & 21 \\
\hline 4 & $\mathrm{CH}_{3}$ & $\mathrm{CH}_{3}$ & $\mathrm{Zn},\left(\mathrm{CH}_{3} \mathrm{O}\right)_{3} \mathrm{~B}$ & 1 & 34 & 60 & 18 \\
\hline
\end{tabular}

respectively. Compounds $\mathbf{2 4 b}$ and $\mathbf{2 6 b}$ were treated similarly to $24 \mathbf{a}$ and $26 \mathbf{a}$ and similar results were obtained. In the case of 25a and $27 \mathbf{a}$, which have tert-butyl ester function, treatments with DBU at room temperature caused the aromatization, but slowly. So the reaction mixtures were heated under reflux for $1 \mathrm{~h}$ (entry 2 and 4), giving 29 and $\mathbf{3 1}$ in 99\% and $99 \%$ yields, respectively. We attributed the lower reactivities of the tert-butyl esters to the steric hindrances around the 2hydrogens, which would be abstracted by DBU. Treatment of the corresponding geometric isomers $\mathbf{2 5 b}$ and $\mathbf{2 7 b}$ in a manner similar to 25a and 27a gave similar results. Similar treatment of the regioisomers $25 \mathbf{c}$ and $25 \mathrm{~d}$ also afforded 29 quantitatively.

The Reformatsky Reactions of $\mathrm{N}$-Tosyl-4-oxoproline Ester and Conversion into the Pyrrole Derivatives It seems impossible that the Horner-Emmons reaction should induce a pyrrole derivative which has a tertiary alkyl group, so we next attempted the Reformatsky reaction. As the usual Reformatsky reaction condition (ketone, bromoacetate, and zinc) often generates side reactions such as an aldol reaction and a dehydration, ${ }^{23}$ addition of trimethyl borate into the reaction system was examined. Unfortunately, methyl ester $\mathbf{5}$ gave complicated mixtures under the conditions employed for 6. As shown in Table 4, when ethyl 2-bromopropionate and ethyl 2-bromoisobutyrate were used (entry 3 and 4), the desired 4-alkylated products 33a, 33b, 34a, and 34b were obtained in $55 \%, 21 \%, 60 \%$, and $18 \%$ yields. Each of 33a and $\mathbf{3 3 b}$ is a mixture of the epimers based on the asymmetric

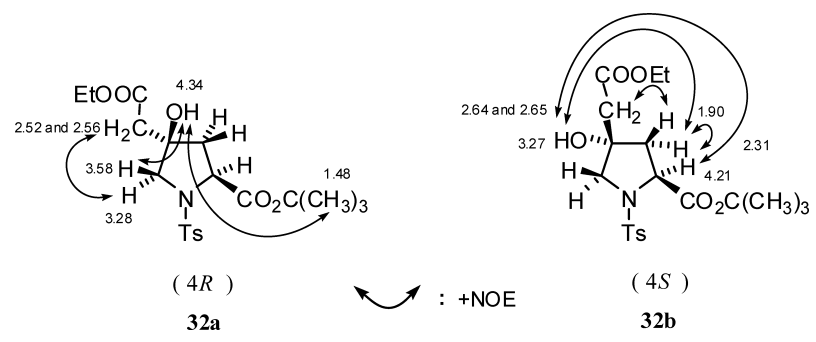

Fig. 4. Selected Decisive NOE Relationships of Compounds 32a and 32b

carbons of side chains; each separation of the epimers was difficult and the ratios of epimers were estimated to be $56: 44$ for 33a, and $66: 34$ for 33b, by ${ }^{1} \mathrm{H}-\mathrm{NMR}$. The same result was obtained when optically active ethyl $(R)$-2-bromopropionate or ethyl $(S)$-2-bromopropionate was employed for the reaction in place of racemic ethyl 2-bromopropionate.

Meanwhile, a complicated mixture was obtained when ethyl bromoacetate was used (entry 1). We therefore attempted the method using $\mathrm{Zn}-\mathrm{Cu}$ couple ${ }^{24)}$ for ethyl bromoacetate (entry 2), and compounds 32a and 32b were obtained in $45 \%$ and $10 \%$ yields.

The stereochemistries of $\mathbf{3 2} \mathbf{a}$ and $\mathbf{3 2} \mathbf{b}$ were determined by differential nuclear Overhauser effect (NOE) experiments of ${ }^{1} \mathrm{H}-\mathrm{NMR}$ (Fig. 4). As for 32a, + NOE was observed between a signal at $\delta 4.34$ assigned to the hydroxy proton and a signal at $\delta 1.48$ assigned to methyl protons of the tert-butyl group. Unfortunately, because the signals of 3-methylene protons overlapped at $\delta 2.16$, no other effective information to deter- 


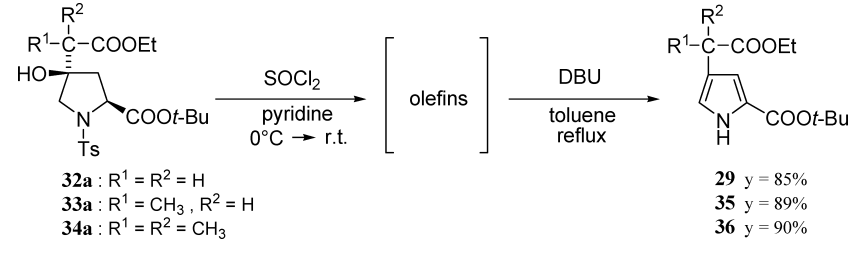

Chart 7

mine the stereochemistry at 4-position was obtained. Therefore, 32a was proved to have a $(2 S, 4 R)$-configuration. For 32b, + NOEs were observed between a signal at $\delta 4.21$ assigned to $2-\mathrm{H}$, a signal at $\delta 3.27$ assigned to the hydroxy proton, and a signal at $\delta 2.31$ assigned to $3-\mathrm{H}_{\alpha}$. + NOEs were also observed between a signal at $\delta 1.90$ assigned to $3-\mathrm{H}_{\beta}$ and signals at $\delta 2.64$ and $\delta 2.65$ assigned to 4-methylene protons. Therefore, $32 \mathbf{b}$ was proved to have a $(2 S, 4 S)$-configuration.

Aromatizations of compounds 32a-34a were done according to those of the Grignard products 10-13, treated with $\mathrm{SOCl}_{2}$-pyridine, then treated with $\mathrm{DBU}$ in refluxing toluene, giving the desired pyrrole derivatives $\mathbf{2 9}, \mathbf{3 5}$, and $\mathbf{3 6}$ in $85 \%, 89 \%$, and $90 \%$ yields, respectively. Enantiomeric compounds $32 \mathbf{b}-\mathbf{3 4} \mathbf{b}$ were also treated similarly to $\mathbf{3 2} \mathbf{a}-$ 34a, and similar results were obtained (Chart 7).

In conclusion, we accomplished the conversion of 4-oxoproline esters to 4-substituted pyrrole-2-carboxylic acid esters.

\section{Experimental}

Melting points were measured with a Yanagimoto micromelting point apparatus and are uncorrected. Specific rotations were determined with a JASCO DIP-370 polarimeter. NMR spectra, except for the amino acids, were recorded in chloroform- $d\left(\mathrm{CDCl}_{3}\right)$ on a JEOL JNM-ECP500 spectrometer using tetramethylsilane as an internal standard. Infrared (IR) spectra were recorded on a Hitachi 270-30 spectrophotometer. Mass spectra (MS) and high resolution mass spectra (HR-MS) were obtained with a JEOL JMSDX300 instrument. TLC was performed on Silica gel $60 \mathrm{~F}_{254}$ plates $(0.25 \mathrm{~mm}$; Merck). Column chromatography was performed on silica gel (Kieselgel 60, 70-230 mesh; Merck). Flash chromatography was performed on silica gel (Silica Gel 60, 230 - 400 mesh; Nacalai Tesque).

Methyl 4-Hydroxypyrrole-2-carboxylate (8) Under an argon atmosphere, compound 5 (1.00 g, $3.36 \mathrm{mmol})$ was suspended in $\mathrm{MeOH}(10 \mathrm{ml})$, and then $28 \% \mathrm{NaOMe}(10 \mathrm{ml})$ was added at room temperature and the mixture was stirred for $1 \mathrm{~h}$. A sufficient amount of carbon dioxide was bubbled into the mixture and the mixture was concentrated under reduced pressure. Water $(25 \mathrm{ml})$ was added to the residue at $0{ }^{\circ} \mathrm{C}$ and the whole was extracted with AcOEt $(25 \mathrm{ml} \times 2)$. The organic layer was washed with water $(25 \mathrm{ml})$, dried over anhydrous $\mathrm{Na}_{2} \mathrm{SO}_{4}$, and concentrated under reduced pressure. The residual brown oil was subjected to column chromatography on silica gel (AcOEt) to give $8(360 \mathrm{mg}, 76 \%)$ as a pale yellow oil. ${ }^{1} \mathrm{H}-\mathrm{NMR}\left(\mathrm{CDCl}_{3}\right) \delta$ $3.83\left(3 \mathrm{H}, \mathrm{s}, \mathrm{CH}_{3}\right), 5.39(1 \mathrm{H}, \mathrm{s}, \mathrm{OH}), 6.51(1 \mathrm{H}, \mathrm{dd}, J=2.8,1.8 \mathrm{~Hz}, 3-\mathrm{H}), 6.58$ $(1 \mathrm{H}, \mathrm{dd}, J=3.0,1.8 \mathrm{~Hz}, 5-\mathrm{H}), 8.84(1 \mathrm{H}, \mathrm{s}, \mathrm{NH}) .{ }^{13} \mathrm{C}-\mathrm{NMR}\left(\mathrm{CDCl}_{3}\right) \delta: 51.63$ (q), 104.47 (d), 108.98 (d), 119.22 (s), $143.81(\mathrm{~s}), 161.79(\mathrm{~s})$. IR $v_{\max }^{\text {neat }} \mathrm{cm}^{-1}$ : 3403, $3134(\mathrm{NH}, \mathrm{OH}), 1685(\mathrm{C}=\mathrm{O})$. HR-MS m/z: 141.0428 (Calcd for $\mathrm{C}_{6} \mathrm{H}_{7} \mathrm{NO}_{3}: 141.0426$ ).

tert-Butyl 4-Hydroxypyrrole-2-carboxylate (9) Under an argon atmosphere, compound $6(1.00 \mathrm{~g}, 2.95 \mathrm{mmol})$ was dissolved in DMSO $(5 \mathrm{ml})$, and then $t$ - $\mathrm{BuOK}(800 \mathrm{mg})$ was added at room temperature and the mixture was stirred for $1 \mathrm{~h}$. A sufficient amount of carbon dioxide was bubbled into the mixture and the mixture was concentrated under reduced pressure. Water $(25 \mathrm{ml})$ was added to the residue at $0^{\circ} \mathrm{C}$ and the whole was extracted with AcOEt $(25 \mathrm{ml} \times 2)$. The organic layer was washed with water $(25 \mathrm{ml})$, dried over anhydrous $\mathrm{Na}_{2} \mathrm{SO}_{4}$, and concentrated under reduced pressure. The residual brown oil was subjected to column chromatography on silica gel [hexane- $\mathrm{Et}_{2} \mathrm{O}(1: 1)$ ] to give $9(325 \mathrm{mg}, 60 \%)$ as a pale yellow oil. ${ }^{1} \mathrm{H}-\mathrm{NMR}$ $\left(\mathrm{CDCl}_{3}\right) \delta: 1.54\left(9 \mathrm{H}, \mathrm{s}, \mathrm{C}\left(\mathrm{CH}_{3}\right)_{3}\right), 5.30(1 \mathrm{H}$, br s, OH), $6.45(1 \mathrm{H}, \mathrm{s}, 3-\mathrm{H})$ $6.53(1 \mathrm{H}, \mathrm{s}, 5-\mathrm{H}), 8.78(1 \mathrm{H}, \mathrm{s}, \mathrm{NH}) .{ }^{13} \mathrm{C}-\mathrm{NMR}\left(\mathrm{CDCl}_{3}\right) \delta: 28.36(\mathrm{q}), 81.17$ (s), $103.97(\mathrm{~d}), 108.04(\mathrm{~d}), 120.96(\mathrm{~s}), 143.66(\mathrm{~s}), 160.83(\mathrm{~s})$. IR $v_{\max }^{\text {neat }} \mathrm{cm}^{-1}$ : 3415, $3315(\mathrm{NH}, \mathrm{OH}), 1681(\mathrm{C}=\mathrm{O})$. HR-MS m/z: 183.0892 (Calcd for $\left.\mathrm{C}_{9} \mathrm{H}_{13} \mathrm{NO}_{3}: 183.0895\right)$.

tert-Butyl $(2 S, 4 R)-4-H y d r o x y-4-m e t h y l-1-p$-toluenesulfonylpyrrolidine-2-carboxylate (10a) and tert-Butyl $(2 S, 4 S)-4-H y d r o x y-4-m e t h y l-1-$ p-toluenesulfonylpyrrolidine-2-carboxylate (10b) Under reduced pressure, powdered $\mathrm{CeCl}_{3} \cdot 7 \mathrm{H}_{2} \mathrm{O}(1.62 \mathrm{~g}, 4.4 \mathrm{mmol})$ was heated at $90^{\circ} \mathrm{C}$ for $1 \mathrm{~h}$, then the temperature was slowly raised and kept at $140{ }^{\circ} \mathrm{C}$ for $2 \mathrm{~h}$. After cooling, the vessel was filled with argon. THF $(15 \mathrm{ml})$ was added and the vessel was irradiated with ultrasonic waves for $2 \mathrm{~h}$. After the suspension was cooled at $-40^{\circ} \mathrm{C}$ under an argon atmosphere, $3 \mathrm{M} \mathrm{CH}_{3} \mathrm{MgBr}$ in $\mathrm{THF}$ $(1.33 \mathrm{ml}, 4.0 \mathrm{mmol})$ was added dropwise and the mixture was stirred for $1.5 \mathrm{~h}$. A solution of compound $6(679 \mathrm{mg}, 2.00 \mathrm{mmol})$ in THF $(3 \mathrm{ml})$ was added dropwise to the suspension prepared as described above and stirred for $1 \mathrm{~h}$. The reaction was quenched with $3 \% \mathrm{CH}_{3} \mathrm{COOH}(13 \mathrm{ml})$. The whole was extracted with AcOEt $(15 \mathrm{ml} \times 3)$ and the organic layer was washed with brine $(15 \mathrm{ml})$, dried over anhydrous $\mathrm{Na}_{2} \mathrm{SO}_{4}$, and concentrated under reduced pressure. The residual white solid was subjected to column chromatography on silica gel [benzene-AcOEt $(10: 1$ then $8: 1)$ ] to give $10 b(472 \mathrm{mg}, 66 \%)$ and then 10a $(130 \mathrm{mg}, 18 \%)$. These compounds were recrystallized from $i$ $\mathrm{Pr}_{2} \mathrm{O}$, respectively.

10a: Colorless prisms, mp $213-214{ }^{\circ} \mathrm{C}($ dec. $),[\alpha]_{\mathrm{D}}^{30}-71.3^{\circ}(c=0.7$, $\left.\mathrm{CHCl}_{3}\right) .{ }^{1} \mathrm{H}-\mathrm{NMR}\left(\mathrm{CDCl}_{3}\right) \delta: 1.35\left(3 \mathrm{H}, \mathrm{s}, \mathrm{CH}_{3}\right), 1.48\left(9 \mathrm{H}, \mathrm{s}, \mathrm{C}\left(\mathrm{CH}_{3}\right)_{3}\right), 1.74$ $\left(1 \mathrm{H}, \mathrm{br}\right.$ s, OH) $1.90\left(1 \mathrm{H}, \mathrm{dd}, J=13.1,9.6 \mathrm{~Hz}, 3-\mathrm{H}_{\beta}\right), 2.25(1 \mathrm{H}$, ddd, $J=13.1$, 7.6, $\left.2.0 \mathrm{~Hz}, 3-\mathrm{H}_{\alpha}\right), 2.41\left(3 \mathrm{H}, \mathrm{s}, \mathrm{Ar}-\mathrm{CH}_{3}\right), 3.34\left(1 \mathrm{H}, \mathrm{d}, J=11.3 \mathrm{~Hz}, 5-\mathrm{H}_{\beta}\right)$, $3.40\left(1 \mathrm{H}, \mathrm{dd}, J=2.0,11.3 \mathrm{~Hz}, 5-\mathrm{H}_{\alpha}\right), 4.33(1 \mathrm{H}, \mathrm{dd}, J=7.6,9.6 \mathrm{~Hz}, 2-\mathrm{H}), 7.30$ $(2 \mathrm{H}, \mathrm{d}, J=8.3 \mathrm{~Hz}, \mathrm{Ar}-\mathrm{H}), 7.79(2 \mathrm{H}, \mathrm{d}, J=8.3 \mathrm{~Hz}, \mathrm{Ar}-\mathrm{H}) .{ }^{13} \mathrm{C}-\mathrm{NMR}\left(\mathrm{CDCl}_{3}\right)$ $\delta: 21.56(\mathrm{q}), 24.15(\mathrm{q}), 27.92(\mathrm{q}), 44.74(\mathrm{t}), 60.82(\mathrm{t}), 61.03$ (d), 76.79 (s), 81.85 (s), 127.81 (d), 129.58 (d), 135.33 (s), 143.66 (s), 171.19 (s). IR $v_{\max }^{\mathrm{KBr}}$ $\mathrm{cm}^{-1}: 3483(\mathrm{OH}), 1728(\mathrm{C}=\mathrm{O})$. MS (FAB) $m / z: 356\left(\mathrm{M}^{+}+1\right)$. Anal. Calcd for $\mathrm{C}_{17} \mathrm{H}_{25} \mathrm{NO}_{5} \mathrm{~S}$ : C, 57.44; H, 7.09; N, 3.94. Found: C, 57.38; H, 6.92; N, 4.01 .

10b: Colorless prisms, mp $127-129^{\circ} \mathrm{C},[\alpha]_{\mathrm{D}}^{30}-51.6^{\circ}\left(c=0.7, \mathrm{CHCl}_{3}\right)$. ${ }^{1} \mathrm{H}-\mathrm{NMR}\left(\mathrm{CDCl}_{3}\right) \delta: 1.29\left(3 \mathrm{H}, \mathrm{s}, \mathrm{CH}_{3}\right), 1.50\left(9 \mathrm{H}, \mathrm{s}, \mathrm{C}\left(\mathrm{CH}_{3}\right)_{3}\right), 1.97(1 \mathrm{H}$, ddd, $\left.J=1.8,2.8,13.8 \mathrm{~Hz}, 3-\mathrm{H}_{\beta}\right), 2.06\left(1 \mathrm{H}, \mathrm{dd}, J=10.3,13.8 \mathrm{~Hz}, 3-\mathrm{H}_{\alpha}\right), 2.44$ $\left(3 \mathrm{H}, \mathrm{s}, \mathrm{Ar}-\mathrm{CH}_{3}\right), 3.06\left(1 \mathrm{H}, \mathrm{d}, J=9.5 \mathrm{~Hz}, 5-\mathrm{H}_{\alpha}\right), 3.46(1 \mathrm{H}, \mathrm{dd}, J=9.5,2.8 \mathrm{~Hz}$, $\left.5-\mathrm{H}_{\beta}\right), 4.16(1 \mathrm{H}, \mathrm{dd}, J=10.3,1.8 \mathrm{~Hz}, 2-\mathrm{H}), 4.29(1 \mathrm{H}, \mathrm{s}, \mathrm{OH}), 7.33(2 \mathrm{H}, \mathrm{d}$, $J=8.1 \mathrm{~Hz}, \mathrm{Ar}-\mathrm{H}), 7.75(2 \mathrm{H}, \mathrm{d}, J=8.1 \mathrm{~Hz}, \mathrm{Ar}-\mathrm{H}) .{ }^{13} \mathrm{C}-\mathrm{NMR}\left(\mathrm{CDCl}_{3}\right) \delta: 21.83$ (q), $23.50(\mathrm{q}), 28.00(\mathrm{q}), 43.90$ (t), $60.72(\mathrm{~d}), 61.34(\mathrm{t}), 77.00(\mathrm{~s}), 83.51(\mathrm{~s})$, $127.96(\mathrm{~d}), 130.03$ (d), $134.76(\mathrm{~s}), 144.21(\mathrm{~s}), 173.53$ (s). IR $v_{\max }^{\mathrm{KBr}} \mathrm{cm}^{-1}$ : $3410(\mathrm{OH}), 1707(\mathrm{C}=\mathrm{O})$. MS (FAB) m/z: $356\left(\mathrm{M}^{+}+1\right)$. Anal. Calcd for $\mathrm{C}_{17} \mathrm{H}_{25} \mathrm{NO}_{5} \mathrm{~S}$ : C, 57.44; H, 7.09; N, 3.94. Found: C, 57.38; H, 6.89; N, 4.14

tert-Butyl (2S,4R)-4-Ethyl-4-hydroxy-1-p-toluenesulfonylpyrrolidine2-carboxylate (11a) and tert-Butyl $(2 S, 4 S)$-4-Ethyl-4-hydroxy-1-p-toluenesulfonylpyrrolidine-2-carboxylate (11b) Compounds 11a (128 mg, $17 \%)$ and $11 b(475 \mathrm{mg}, 64 \%)$ were obtained from $6(679 \mathrm{mg}, 2.00 \mathrm{mmol})$ in a manner similar to that described for 10, but using $2 \mathrm{M} \mathrm{EtMgCl}$ in $\mathrm{Et}_{2} \mathrm{O}$ $(2.0 \mathrm{ml}, 4.0 \mathrm{mmol})$ and a reaction time of $1.5 \mathrm{~h}$. These compounds were recrystallized from $i-\mathrm{Pr}_{2} \mathrm{O}$, respectively.

11a: Colorless prisms, mp $114-115^{\circ} \mathrm{C},[\alpha]_{\mathrm{D}}^{30}-77.2^{\circ}\left(c=0.6, \mathrm{CHCl}_{3}\right)$. ${ }^{1} \mathrm{H}-\mathrm{NMR}\left(\mathrm{CDCl}_{3}\right) \delta: 0.92\left(3 \mathrm{H}, \mathrm{t}, J=7.6 \mathrm{~Hz}, \mathrm{CH}_{2} \mathrm{CH}_{3}\right), 1.48(9 \mathrm{H}, \mathrm{s}$, $\left.\mathrm{C}\left(\mathrm{CH}_{3}\right)_{3}\right), 1.61\left(2 \mathrm{H}, \mathrm{q}, J=7.6 \mathrm{~Hz}, \mathrm{CH}_{2} \mathrm{CH}_{3}\right), 1.85(1 \mathrm{H}, \mathrm{dd}, J=13.0,9.6 \mathrm{~Hz}$, $\left.3-\mathrm{H}_{\alpha}\right), 1.85(1 \mathrm{H}, \mathrm{s}, \mathrm{OH}), 2.22\left(1 \mathrm{H}, \mathrm{ddd}, J=13.0,7.5,1.8 \mathrm{~Hz}, 3-\mathrm{H}_{\beta}\right), 2.41$ $\left(3 \mathrm{H}, \mathrm{s}, \mathrm{Ar}-\mathrm{CH}_{3}\right), 3.33\left(1 \mathrm{H}, \mathrm{d}, J=11.5 \mathrm{~Hz}, 5-\mathrm{H}_{\beta}\right), 3.39(1 \mathrm{H}, \mathrm{dd}, J=11.5$, $\left.1.8 \mathrm{~Hz}, 5-\mathrm{H}_{\alpha}\right), 4.32(1 \mathrm{H}, \mathrm{dd}, J=9.6,7.5 \mathrm{~Hz}, 2-\mathrm{H}), 7.31(2 \mathrm{H}, \mathrm{d}, J=8.4 \mathrm{~Hz}, \mathrm{Ar}-$ $\mathrm{H}), 7.80(2 \mathrm{H}, \mathrm{d}, J=8.4 \mathrm{~Hz}, \mathrm{Ar}-\mathrm{H}) .{ }^{13} \mathrm{C}-\mathrm{NMR}\left(\mathrm{CDCl}_{3}\right) \delta: 8.48(\mathrm{q}), 21.56(\mathrm{q})$, $27.92(\mathrm{q}), 30.52(\mathrm{t}), 42.84(\mathrm{t}), 59.80(\mathrm{t}), 61.00(\mathrm{~d}), 79.81(\mathrm{~s}), 81.82(\mathrm{~s})$, 127.80 (d), 129.57 (d), 135.34 (s), $143.64(\mathrm{~s}), 171.35$ (s). IR $v_{\max }^{\mathrm{KBr}} \mathrm{cm}^{-1}$ : $3502(\mathrm{OH}), 1751(\mathrm{C}=\mathrm{O})$. MS (FAB) $m / z: 370\left(\mathrm{M}^{+}+1\right)$. Anal. Calcd for $\mathrm{C}_{18} \mathrm{H}_{27} \mathrm{NO}_{5} \mathrm{~S}$ : C, 58.51; H, 7.37; N, 3.79. Found: C, 58.56; H, 7.24; N, 3.78.

11b: Colorless prisms, mp $126-127^{\circ} \mathrm{C},[\alpha]_{\mathrm{D}}^{26}-48.9^{\circ}\left(c=1.0, \mathrm{CHCl}_{3}\right)$. ${ }^{1} \mathrm{H}-\mathrm{NMR}\left(\mathrm{CDCl}_{3}\right) \delta: 0.94\left(3 \mathrm{H}, \mathrm{t}, J=7.4 \mathrm{~Hz}, \mathrm{CH}_{2} \mathrm{CH}_{3}\right), 1.50(9 \mathrm{H}, \mathrm{s}$, $\left.\mathrm{C}\left(\mathrm{CH}_{3}\right)_{3}\right), 1.56\left(2 \mathrm{H}, \mathrm{q}, J=7.4 \mathrm{~Hz}, \mathrm{CH}_{2} \mathrm{CH}_{3}\right), 1.94(1 \mathrm{H}, \mathrm{dt}, J=13.7,1.6 \mathrm{~Hz}, 3-$ $\left.\mathrm{H}_{\alpha}\right), 2.02\left(1 \mathrm{H}, \mathrm{dd}, J=13.7,10.3 \mathrm{~Hz}, 3-\mathrm{H}_{\beta}\right), 2.44\left(3 \mathrm{H}, \mathrm{s}, \mathrm{Ar}-\mathrm{CH}_{3}\right), 3.05(1 \mathrm{H}$, d, $\left.J=9.4 \mathrm{~Hz}, 5-\mathrm{H}_{\beta}\right), 3.45\left(1 \mathrm{H}, \mathrm{dd}, J=9.4,1.6 \mathrm{~Hz}, 5-\mathrm{H}_{\alpha}\right), 4.17(1 \mathrm{H}, \mathrm{s}, \mathrm{OH})$, $4.19(1 \mathrm{H}, \mathrm{d}, J=1.6 \mathrm{~Hz}, 2-\mathrm{H}), 7.33(2 \mathrm{H}, \mathrm{d}, J=8.1 \mathrm{~Hz}, \mathrm{Ar}-\mathrm{H}), 7.76(2 \mathrm{H}, \mathrm{d}$, $J=8.1 \mathrm{~Hz}, \mathrm{Ar}-\mathrm{H}) .{ }^{13} \mathrm{C}-\mathrm{NMR}\left(\mathrm{CDCl}_{3}\right) \delta: 8.67(\mathrm{q}), 21.56(\mathrm{q}), 27.81(\mathrm{q}), 30.32$ (t), 42.05 (t), $59.96(\mathrm{t}), 60.31$ (d), 79.57 (s), 83.17 (s), 127.68 (d), 129.76 (d), $134.57(\mathrm{~s}), 143.91$ (s), 173.29 (s). IR $v_{\max }^{\text {neat }} \mathrm{cm}^{-1}: 3465(\mathrm{OH}), 1720$ $(\mathrm{C}=\mathrm{O})$. MS (FAB) $m / z$ : $370\left(\mathrm{M}^{+}+1\right)$. Anal. Calcd for $\mathrm{C}_{18} \mathrm{H}_{27} \mathrm{NO}_{5} \mathrm{~S}: \mathrm{C}$, 58.51; H, 7.37; N, 3.79. Found: C, 58.61; H, 7.31; N, 3.74.

tert-Butyl (2S,4R)-4-Butyl-4-hydroxy-1-p-toluenesulfonylpyrrolidine2-carboxylate (12a) and tert-Butyl (2S,4S)-4-Butyl-4-hydroxy-1-p-toluenesulfonylpyrrolidine-2-carboxylate (12b) Compounds 12a (136 mg, 
$17 \%)$ and $12 \mathrm{~b}(588 \mathrm{mg}, 74 \%)$ were obtained from 6 (679 $\mathrm{mg}, 2.0 \mathrm{mmol})$ in a manner similar to that described for 10, but using $2 \mathrm{M} \mathrm{BuMgCl}$ in $\mathrm{Et}_{2} \mathrm{O}$ $(2.0 \mathrm{ml}, 4.0 \mathrm{mmol})$, a reaction time of $2 \mathrm{~h}$, and column chromatography on silica gel [benzene-AcOEt $(15: 1$ then $10: 1)]$. These compounds were recrystallized from $i-\mathrm{Pr}_{2} \mathrm{O}$, respectively.

12a: Colorless oil, $[\alpha]_{\mathrm{D}}^{26}-45.8^{\circ}\left(c=0.8, \mathrm{CHCl}_{3}\right)$. ${ }^{1} \mathrm{H}-\mathrm{NMR}\left(\mathrm{CDCl}_{3}\right) \delta$ : $0.88\left(3 \mathrm{H}, \mathrm{t}, J=7.1 \mathrm{~Hz},\left(\mathrm{CH}_{2}\right)_{3} \mathrm{CH}_{3}\right), 1.23-1.30\left(2 \mathrm{H}, \mathrm{m}, \mathrm{CH}_{2} \mathrm{CH}_{2} \mathrm{CH}_{2} \mathrm{CH}_{3}\right)$ $1.48\left(11 \mathrm{H}, \quad \mathrm{s}, \quad \mathrm{C}\left(\mathrm{CH}_{3}\right)_{3}\right.$ and $\left.\mathrm{C}_{2}\left(\mathrm{CH}_{2}\right)_{2} \mathrm{CH}_{3}\right), 1.51-1.58(4 \mathrm{H}, \mathrm{m}$, $\left.\mathrm{CH}_{2} \mathrm{CH}_{2} \mathrm{CH}_{2} \mathrm{CH}_{3}\right), 1.78(1 \mathrm{H}, \mathrm{s}, \mathrm{OH}), 1.86\left(1 \mathrm{H}, \mathrm{dd}, J=13.1,9.9 \mathrm{~Hz}, 3-\mathrm{H}_{\alpha}\right)$, $2.22\left(1 \mathrm{H}, \mathrm{ddd}, J=13.1,7.3,1.8 \mathrm{~Hz}, 3-\mathrm{H}_{\beta}\right), 2.42\left(3 \mathrm{H}, \mathrm{s}, \mathrm{Ar}_{-} \mathrm{CH}_{3}\right), 3.33(1 \mathrm{H}, \mathrm{d}$ $\left.J=11.5 \mathrm{~Hz}, 5-\mathrm{H}_{\alpha}\right), 3.40\left(1 \mathrm{H}, \mathrm{dd}, J=11.5,1.8 \mathrm{~Hz}, 5-\mathrm{H}_{\beta}\right), 4.32(1 \mathrm{H}, \mathrm{dd}$ $J=9.9,7.3 \mathrm{~Hz}, 2-\mathrm{H}), 7.31(2 \mathrm{H}, \mathrm{d}, J=8.3 \mathrm{~Hz}, \mathrm{Ar}-\mathrm{H}), 7.80(2 \mathrm{H}, \mathrm{d}, J=8.3 \mathrm{~Hz}$ Ar-H). ${ }^{13} \mathrm{C}-\mathrm{NMR}\left(\mathrm{CDCl}_{3}\right) \delta: 13.93(\mathrm{q}), 21.57(\mathrm{q}), 22.96(\mathrm{t}), 26.39(\mathrm{t}), 27.93$ $(\mathrm{q}), 37.47(\mathrm{t}), 43.31(\mathrm{t}), 60.05(\mathrm{t}), 60.89(\mathrm{~d}), 79.55(\mathrm{~s}), 81.82(\mathrm{~s}), 127.81(\mathrm{~d})$, $129.58(\mathrm{~d}), 135.37(\mathrm{~s}), 143.66(\mathrm{~s}), 171.29(\mathrm{~s})$. IR $v_{\max }^{\text {neat }} \mathrm{cm}^{-1}: 3485(\mathrm{OH})$, $1730(\mathrm{C}=\mathrm{O})$. MS (FAB) $m / z: 398\left(\mathrm{M}^{+}+1\right)$.

12b: Colorless needles, mp $116-117^{\circ} \mathrm{C},[\alpha]_{\mathrm{D}}^{30}-41.8^{\circ}\left(c=1.0, \mathrm{CHCl}_{3}\right)$ ${ }^{1} \mathrm{H}-\mathrm{NMR}\left(\mathrm{CDCl}_{3}\right) \delta: 0.87\left(3 \mathrm{H}, \mathrm{t}, J=7.1 \mathrm{~Hz},\left(\mathrm{CH}_{2}\right)_{3} \mathrm{CH}_{3}\right), 1.25-1.31(2 \mathrm{H}$, $\left.\mathrm{m}, \mathrm{CH}_{2} \mathrm{CH}_{2} \mathrm{CH}_{2} \mathrm{CH}_{3}\right), 1.32-1.39\left(2 \mathrm{H}, \mathrm{m}, \mathrm{CH}_{2} \mathrm{CH}_{2} \mathrm{CH}_{2} \mathrm{CH}_{3}\right), 1.50(11 \mathrm{H}, \mathrm{s}$ $\mathrm{C}\left(\mathrm{CH}_{3}\right)_{3}$ and $\left.\mathrm{CH}_{2}\left(\mathrm{CH}_{2}\right)_{2} \mathrm{CH}_{3}\right), 1.95\left(1 \mathrm{H}\right.$, ddd, $\left.J=13.7,1.8,1.6 \mathrm{~Hz}, 3-\mathrm{H}_{\beta}\right)$, $2.02\left(1 \mathrm{H}, \mathrm{dd}, J=13.7,10.3 \mathrm{~Hz}, 3-\mathrm{H}_{\alpha}\right), 2.44\left(3 \mathrm{H}, \mathrm{s}, \mathrm{Ar}-\mathrm{CH}_{3}\right), 3.04(1 \mathrm{H}, \mathrm{d}$ $\left.J=9.5 \mathrm{~Hz}, 5-\mathrm{H}_{\alpha}\right), 3.45\left(1 \mathrm{H}, \mathrm{dd}, J=9.5,1.6 \mathrm{~Hz}, 5-\mathrm{H}_{\beta}\right), 4.16(1 \mathrm{H}, \mathrm{dd}, J=10.3$, $1.8 \mathrm{~Hz}, 2-\mathrm{H}), 4.18(1 \mathrm{H}, \mathrm{s}, \mathrm{OH}), 7.32(1 \mathrm{H}, \mathrm{d}, J=8.3 \mathrm{~Hz}, \mathrm{Ar}-\mathrm{H}), 7.75(2 \mathrm{H}, \mathrm{d}$ $J=8.3 \mathrm{~Hz}$, Ar-H). ${ }^{13} \mathrm{C}-\mathrm{NMR}\left(\mathrm{CDCl}_{3}\right) \delta: 13.95$ (q), 21.57 (q), 23.05 (q), $26.52(\mathrm{t}), 27.81(\mathrm{q}), 37.29(\mathrm{t}), 42.46(\mathrm{t}), 60.18(\mathrm{t}), 60.21(\mathrm{~d}), 79.28(\mathrm{~s}), 83.21$ (s), 127.70 (d), 129.76 (d), 134.55 (s), 143.91 (s), 173.29 (s). IR $v_{\max }^{\mathrm{KBr}} \mathrm{cm}^{-1}$ : $3467(\mathrm{OH}), 1718(\mathrm{C}=\mathrm{O})$. MS (FAB) $m / z: 398\left(\mathrm{M}^{+}+1\right)$. Anal. Calcd for $\mathrm{C}_{18} \mathrm{H}_{27} \mathrm{NO}_{5} \mathrm{~S}: \mathrm{C}, 60.43 ; \mathrm{H}, 7.86 ; \mathrm{N}, 3.52$. Found: $\mathrm{C}, 60.51 ; \mathrm{H}, 7.71 ; \mathrm{N}, 3.38$.

tert-Butyl $(2 S, 4 R)$-4-Hydroxy-4-phenyl-1-p-toluenesulfonylpyrrolidine2-carboxylate (13) Compound $13(762 \mathrm{mg}, 91 \%)$ was obtained from 6 $(679 \mathrm{mg}, 2.0 \mathrm{mmol})$ in a manner similar to that described for 10, but using $1 \mathrm{M} \mathrm{PhMgBr}$ in THF $(4.0 \mathrm{ml}, 4.0 \mathrm{mmol})$, a reaction time of $2 \mathrm{~h}$, and column chromatography on silica gel [benzene- $\left.\mathrm{Et}_{2} \mathrm{O}(40: 1)\right]$. The resulting white solid was recrystallized from $i$-PrOH to give colorless needles, 166$168^{\circ} \mathrm{C},[\alpha]_{\mathrm{D}}^{29}-80.9^{\circ}\left(c=1.0, \mathrm{CHCl}_{3}\right) .{ }^{1} \mathrm{H}-\mathrm{NMR}\left(\mathrm{CDCl}_{3}\right) \delta: 1.54(9 \mathrm{H}, \mathrm{s}$ $\left.\mathrm{C}\left(\mathrm{CH}_{3}\right)_{3}\right), 2.25\left(1 \mathrm{H}, \mathrm{dd}, J=14.0,1.6 \mathrm{~Hz}, 3-\mathrm{H}_{\beta}\right), 2.44\left(3 \mathrm{H}, \mathrm{s}, \mathrm{Ar}-\mathrm{CH}_{3}\right), 2.55$ $\left(1 \mathrm{H}, \mathrm{dd}, J=14.0,10.6 \mathrm{~Hz}, 3-\mathrm{H}_{\alpha}\right), 3.44\left(1 \mathrm{H}, \mathrm{d}, J=9.6 \mathrm{~Hz}, 5-\mathrm{H}_{\alpha}\right), 3.71(1 \mathrm{H}$, dd, $\left.J=9.6,1.6 \mathrm{~Hz}, 5-\mathrm{H}_{\beta}\right), 4.35(1 \mathrm{H}, \mathrm{dd}, J=10.6,1.6 \mathrm{~Hz}, 2-\mathrm{H}), 4.75(1 \mathrm{H}, \mathrm{s}$, $\mathrm{OH}), 7.30(5 \mathrm{H}, \mathrm{m}, \mathrm{Ph}), 7.38(2 \mathrm{H}, \mathrm{d}, J=7.6 \mathrm{~Hz}, \mathrm{Ar}-\mathrm{H}), 7.80(2 \mathrm{H}, \mathrm{d}$ $J=7.6 \mathrm{~Hz}, \mathrm{Ar}-\mathrm{H}) .{ }^{13} \mathrm{C}-\mathrm{NMR}\left(\mathrm{CDCl}_{3}\right) \delta: 21.59(\mathrm{q}), 27.85(\mathrm{q}), 44.01(\mathrm{t}), 60.27$ (d), $61.67(\mathrm{t}), 79.91(\mathrm{~s}), 83.51(\mathrm{~s}), 125.24(\mathrm{~d}), 127.78(\mathrm{~d}), 127.91(\mathrm{~d}), 128.46$ (d), $129.84(\mathrm{~s}), 134.44$ (d), $140.18(\mathrm{~s}), 144.08(\mathrm{~s}), 173.12$ (s). IR $v_{\max }^{\mathrm{KBr}} \mathrm{cm}^{-1}$. $3398(\mathrm{OH}), 1703(\mathrm{C}=\mathrm{O})$. MS $(\mathrm{FAB}) m / z: 418\left(\mathrm{M}^{+}+1\right)$. Anal. Calcd for $\mathrm{C}_{22} \mathrm{H}_{27} \mathrm{NO}_{5} \mathrm{~S}$ : C, 63.29; H, 6.52; N, 3.35. Found: $\mathrm{C}, 63.16 ; \mathrm{H}, 6.45 ; \mathrm{N}, 3.37$.

Methyl 4-Methylpyrrole-2-carboxylate (4) Thionyl chloride $(0.61 \mathrm{ml}$, $7.9 \mathrm{mmol})$ was added dropwise to $\mathrm{MeOH}(5 \mathrm{ml})$ at $-10^{\circ} \mathrm{C}$. After $30 \mathrm{~min}$, compound 10b $(200 \mathrm{mg}, 0.56 \mathrm{mmol})$ was added to the solution. The whole was stirred at room temperature for $24 \mathrm{~h}$ and the reaction mixture was concentrated under reduced pressure. $\mathrm{MeOH}(15 \mathrm{ml})$ was added to the residue and the solution was concentrated under reduced pressure. This operation was repeated 3 times. The residual oil was dissolved in $\mathrm{MeOH}(3 \mathrm{ml})$ and $28 \% \mathrm{NaOMe}$ solution $(3 \mathrm{ml})$ was added. Then the mixture was stirred at room temperature for $1.5 \mathrm{~h}$. A sufficient amount of carbon dioxide was bubbled into the mixture and the mixture was concentrated under reduced pressure. Water $(20 \mathrm{ml})$ was added to the residue at $0{ }^{\circ} \mathrm{C}$ and the whole was extracted with AcOEt $(20 \mathrm{ml} \times 2)$. The organic layer was washed with brine $(20 \mathrm{ml})$, dried over anhydrous $\mathrm{Na}_{2} \mathrm{SO}_{4}$, and concentrated under reduced pressure. The residue was subjected to column chromatography on silica gel (AcOEt) to give $4(43 \mathrm{mg}, 56 \%)$ as a white solid. It was recrystallized from cyclohexane to give colorless needles, mp $73-74{ }^{\circ} \mathrm{C}$, (lit. ${ }^{25)} \mathrm{mp} 73-74{ }^{\circ} \mathrm{C}$ ). ${ }^{1} \mathrm{H}-\mathrm{NMR}\left(\mathrm{CDCl}_{3}\right) \delta: 2.10\left(3 \mathrm{H}, \mathrm{s}, \mathrm{CH}_{3}\right), 3.84\left(3 \mathrm{H}, \mathrm{s}, \mathrm{OCH}_{3}\right), 6.72(2 \mathrm{H}, \mathrm{s}, 3-$ $\mathrm{H}$ and $5-\mathrm{H}), 9.20\left(1 \mathrm{H}\right.$, br s, NH). ${ }^{13} \mathrm{C}-\mathrm{NMR}\left(\mathrm{CDCl}_{3}\right) \delta: 11.67(\mathrm{q}), 51.36(\mathrm{q})$ 116.12 (d), $120.94(\mathrm{~s}), 121.50$ (d), 122.16 (s), 161.79 (s). IR $v_{\max }^{\text {neat }} \mathrm{cm}^{-1}$ : $3283(\mathrm{NH}), 1673(\mathrm{C}=\mathrm{O})$. MS $m / z: 139\left(\mathrm{M}^{+}\right)$.

Methyl 4-Ethylpyrrole-2-carboxylate (14) Compound 14 (47 mg, $55 \%)$ was obtained from $11 \mathrm{~b}(207 \mathrm{mg}, 0.56 \mathrm{mmol})$ in a manner similar to that described for $\mathbf{4}$ but with column chromatography on silica gel [hexane-AcOEt $(1: 1)]$. It was recrystallized from hexane to give colorless

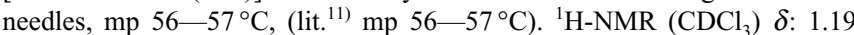
$\left(3 \mathrm{H}, \mathrm{t}, J=7.6 \mathrm{~Hz}, \mathrm{CH}_{2} \mathrm{C}_{3}\right), 2.50\left(2 \mathrm{H}, \mathrm{q}, J=7.6 \mathrm{~Hz}, \mathrm{C}_{2} \mathrm{CH}_{3}\right), 3.84(3 \mathrm{H}, \mathrm{s}$, $\left.\mathrm{CO}_{2} \mathrm{CH}_{3}\right), 6.76(2 \mathrm{H}, \mathrm{s}, 3-\mathrm{H}$ and $5-\mathrm{H}), 8.87(1 \mathrm{H}, \mathrm{br}, \mathrm{NH}) .{ }^{13} \mathrm{C}-\mathrm{NMR}$ $\left(\mathrm{CDCl}_{3}\right) \delta: 15.17(\mathrm{q}), 19.86(\mathrm{t}), 51.35(\mathrm{q}), 114.56(\mathrm{~d}), 120.08(\mathrm{~d}), 122.23(\mathrm{~s})$ $128.42(\mathrm{~s}), 161.57(\mathrm{~s})$. IR $v_{\max }^{\mathrm{KBr}} \mathrm{cm}^{-1}: 3292(\mathrm{NH}), 1685(\mathrm{C}=\mathrm{O})$. MS $m / z: 153$ $\left(\mathrm{M}^{+}\right)$
Methyl 4-Butylpyrrole-2-carboxylate (15) Compound 15 (56 mg, $55 \%)$ was obtained from $12 \mathrm{~b}(223 \mathrm{mg}, 0.56 \mathrm{mmol})$ in a manner similar to that described for 4 but with column chromatography on silica gel [hexane-AcOEt $(2: 1)]$. It was recrystallized from hexane to give colorless

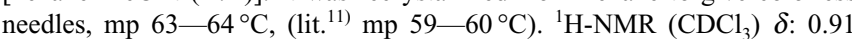
$\left(3 \mathrm{H}, \mathrm{t}, J=7.3 \mathrm{~Hz},\left(\mathrm{CH}_{2}\right)_{3} \mathrm{CH}_{3}\right), 1.31-1.39\left(2 \mathrm{H}, \mathrm{m},\left(\mathrm{CH}_{2}\right)_{2} \mathrm{CH}_{2} \mathrm{CH}_{3}\right), 1.51-$ $1.57\left(2 \mathrm{H}, \mathrm{m}, \mathrm{CH}_{2} \mathrm{CH}_{2} \mathrm{CH}_{2} \mathrm{CH}_{3}\right), 2.46(2 \mathrm{H}, \mathrm{t}, J=7.3 \mathrm{~Hz}$, benzylic $\mathrm{H}), 3.83$ $\left(3 \mathrm{H}, \mathrm{s}, \mathrm{CO}_{2} \mathrm{CH}_{3}\right), 6.73(1 \mathrm{H}, \mathrm{s}, 3-\mathrm{H}), 6.75(1 \mathrm{H}, \mathrm{s}, 5-\mathrm{H}), 8.95(1 \mathrm{H}, \mathrm{br}, \mathrm{NH})$ ${ }^{13} \mathrm{C}-\mathrm{NMR}\left(\mathrm{CDCl}_{3}\right) \delta: 13.92(\mathrm{q}), 22.30(\mathrm{t}), 26.36(\mathrm{t}), 33.12(\mathrm{t}), 51.34(\mathrm{q})$ 115.04 (d), 120.65 (d), 122.14 (s), 126.81 (s), 161.63 (s). IR $v_{\max }^{\mathrm{KBr}} \mathrm{cm}^{-1}$ $3300(\mathrm{NH}), 1693(\mathrm{C}=\mathrm{O})$. MS $m / z: 181\left(\mathrm{M}^{+}\right)$.

Methyl 4-Phenylpyrrole-2-carboxylate (16) Compound 16 (89 mg, $79 \%)$ was obtained from $\mathbf{1 3}(234 \mathrm{mg}, 0.56 \mathrm{mmol})$ in a manner similar to that described for 4. It was recrystallized from $i-\mathrm{Pr}_{2} \mathrm{O}$ to give colorless prisms, $\mathrm{mp} 197-198^{\circ} \mathrm{C}$, $\left(\right.$ lit. $\left.^{26)} \mathrm{mp} 176-180^{\circ} \mathrm{C}\right) .{ }^{1} \mathrm{H}-\mathrm{NMR}\left(\mathrm{CDCl}_{3}\right) \delta: 3.89(3 \mathrm{H}, \mathrm{s}$, $\left.\mathrm{OCH}_{3}\right), 7.20-7.25(3 \mathrm{H}, \mathrm{m}, \mathrm{Ar}-\mathrm{H}), 7.36(2 \mathrm{H}, \mathrm{m}, \mathrm{Ar}-\mathrm{H}), 7.52(2 \mathrm{H}, \mathrm{m}, 3-\mathrm{H}$ and 5-H), $9.17\left(1 \mathrm{H}\right.$, br s, NH). ${ }^{13} \mathrm{C}-\mathrm{NMR}\left(\mathrm{CDCl}_{3}\right) \delta: 51.61(\mathrm{q}), 112.55(\mathrm{~d})$ 119.51 (d), 123.42 (s), 125.31 (d), 126.34 (d), 126.94 (s), 128.79 (d), 134.48 (s), $161.56(\mathrm{~s})$. IR $v_{\mathrm{max}}^{\mathrm{KBr}} \mathrm{cm}^{-1}: 3280(\mathrm{NH}), 1677(\mathrm{C}=\mathrm{O})$. MS $m / z: 201\left(\mathrm{M}^{+}\right)$ Anal. Calcd for $\mathrm{C}_{12} \mathrm{H}_{11} \mathrm{NO}_{2}: \mathrm{C}, 71.63 ; \mathrm{H}, 5.51 ; \mathrm{N}, 6.96$. Found: $\mathrm{C}, 71.46 ; \mathrm{H}$, $5.64 ; \mathrm{N}, 6.97$

tert-Butyl 4-Methylpyrrole-2-carboxylate (17) Thionyl chloride $(4.0 \mathrm{ml}, 55 \mathrm{mmol})$ was added dropwise to a solution of compound $\mathbf{1 0 b}$ $(200 \mathrm{mg}, 0.56 \mathrm{mmol})$ in pyridine $(12 \mathrm{ml})$ and the whole was stirred at room temperature for $2 \mathrm{~h}$. The reaction mixture was concentrated under reduced pressure. Toluene $(12 \mathrm{ml})$ was added and the solution was concentrated under reduced pressure. This operation was repeated once. Water $(20 \mathrm{ml})$ was added to the residue and the whole was extracted with benzene $(20 \mathrm{ml} \times 2)$. The organic layer was washed with brine $(20 \mathrm{ml})$, dried over anhydrous $\mathrm{Na}_{2} \mathrm{SO}_{4}$, and concentrated under reduced pressure. The residue was dissolved in toluene $(6 \mathrm{ml})$ and $\mathrm{DBU}(0.5 \mathrm{ml}, 3.3 \mathrm{mmol})$, and the solution was refluxed for $12 \mathrm{~h}$. The mixture was concentrated under reduced pressure and the residue was subjected to column chromatography on silica gel [hexane-AcOEt (10:1)] to give $\mathbf{1 7}(79 \mathrm{mg}, 77 \%)$ as a white solid. It was recrystallized from hexane to give colorless prisms, mp $113-114{ }^{\circ} \mathrm{C} .{ }^{1} \mathrm{H}-$ NMR $\left(\mathrm{CDCl}_{3}\right) \delta: 1.59\left(9 \mathrm{H}, \mathrm{s}, \mathrm{C}\left(\mathrm{CH}_{3}\right)_{3}\right), 2.10\left(3 \mathrm{H}, \mathrm{s}, \mathrm{CH}_{3}\right), 6.66(1 \mathrm{H}, \mathrm{s}, 3-$ $\mathrm{H}), 6.68(1 \mathrm{H}, \mathrm{s}, 5-\mathrm{H}), 9.08(1 \mathrm{H}, \mathrm{br} \mathrm{s}, \mathrm{NH}) .{ }^{13} \mathrm{C}-\mathrm{NMR}\left(\mathrm{CDCl}_{3}\right) \delta: 11.69(\mathrm{q})$ 28.39 (q), 80.57 (s), 115.40 (d), 120.55 (d), 120.64 (s), 123.98 (s), 160.82 (s). IR $v_{\mathrm{mar}}^{\mathrm{KBr}} \mathrm{cm}^{-1}: 3323(\mathrm{NH}), 1676(\mathrm{C}=\mathrm{O})$. MS (FAB) $\mathrm{m} / \mathrm{z}: 182\left(\mathrm{M}^{+}+1\right)$. Anal. Calcd for $\mathrm{C}_{10} \mathrm{H}_{15} \mathrm{NO}_{2}$ : C, 66.27; $\mathrm{H}, 8.34 ; \mathrm{N}, 7.73$. Found: $\mathrm{C}, 66.36 ; \mathrm{H}$, $8.40 ; \mathrm{N}, 7.70$.

tert-Butyl 4-Ethylpyrrole-2-carboxylate (18) Compound 18 (90 mg, $82 \%)$ was obtained from $11 \mathrm{~b}(207 \mathrm{mg}, 0.56 \mathrm{mmol})$ in a manner similar to that described for 17. It was recrystallized from hexane to give colorless prisms, mp $75-76{ }^{\circ} \mathrm{C} .{ }^{1} \mathrm{H}-\mathrm{NMR}\left(\mathrm{CDCl}_{3}\right) \delta: 1.19(3 \mathrm{H}, \mathrm{t}, J=7.6 \mathrm{~Hz}$, $\left.\mathrm{CH}_{2} \mathrm{CH}_{3}\right), 1.55\left(9 \mathrm{H}, \mathrm{s}, \mathrm{C}\left(\mathrm{CH}_{3}\right)_{3}\right), 2.49\left(2 \mathrm{H}, \mathrm{q}, J=7.6 \mathrm{~Hz}, \mathrm{CH}_{2} \mathrm{CH}_{3}\right), 6.69$ $(2 \mathrm{H}, \mathrm{s}, 3-\mathrm{H}$ and $5-\mathrm{H}), 9.03(1 \mathrm{H}, \mathrm{br}, \mathrm{NH}) \cdot{ }^{13} \mathrm{C}-\mathrm{NMR}\left(\mathrm{CDCl}_{3}\right) \delta: 15.28(\mathrm{q})$ $19.91(\mathrm{t}), 28.40(\mathrm{q}), 80.57$ (s), 113.89 (d), 119.41 (d), $123.97(\mathrm{~s}), 128.07$ (s), 160.79 (s). IR $v_{\max }^{\mathrm{KBr}} \mathrm{cm}^{-1}: 3319(\mathrm{NH}), 1672(\mathrm{C}=\mathrm{O})$. MS (FAB) $\mathrm{m} / \mathrm{z}: 196$ $\left(\mathrm{M}^{+}+1\right)$. Anal. Calcd for $\mathrm{C}_{11} \mathrm{H}_{17} \mathrm{NO}_{2}: \mathrm{C}, 67.66 ; \mathrm{H}, 8.78 ; \mathrm{N}, 7.17$. Found: $\mathrm{C}$, $67.89 ; \mathrm{H}, 8.72 ; \mathrm{N}, 7.14$.

tert-Butyl 4-Butylpyrrole-2-carboxylate (19) Compound 19 (75 mg, $60 \%)$ was obtained from $12 \mathrm{~b}(223 \mathrm{mg}, 0.56 \mathrm{mmol})$ in a manner similar to that described for 17. It was recrystallized from hexane to give colorless prisms, mp $70-71{ }^{\circ} \mathrm{C} .{ }^{1} \mathrm{H}-\mathrm{NMR}\left(\mathrm{CDCl}_{3}\right) \delta: 0.92(3 \mathrm{H}, \mathrm{t}, J=7.4 \mathrm{~Hz}$, $\left.\left(\mathrm{CH}_{2}\right)_{3} \mathrm{CH}_{3}\right), 1.33\left(2 \mathrm{H}, \mathrm{m},\left(\mathrm{CH}_{2}\right)_{2} \mathrm{CH}_{2} \mathrm{CH}_{3}\right), 1.55\left(11 \mathrm{H}, \mathrm{s}, \mathrm{C}\left(\mathrm{CH}_{3}\right)_{3}\right.$ and $\left.\mathrm{CH}_{2} \mathrm{CH}_{2} \mathrm{CH}_{2} \mathrm{CH}_{3}\right), 2.45\left(2 \mathrm{H}, \mathrm{t}, J=7.7 \mathrm{~Hz}, \mathrm{CH}_{2}\left(\mathrm{CH}_{2}\right)_{2} \mathrm{CH}_{3}\right), 6.67(2 \mathrm{H}, \mathrm{s}, 3-\mathrm{H}$ and $5-\mathrm{H}), 8.87(1 \mathrm{H}, \mathrm{brs}, \mathrm{NH}) .{ }^{13} \mathrm{C}-\mathrm{NMR}\left(\mathrm{CDCl}_{3}\right) \delta: 13.93(\mathrm{q}), 22.36(\mathrm{t})$, $26.43(\mathrm{t}), 28.41(\mathrm{q}), 33.22(\mathrm{t}), 80.56(\mathrm{~s}), 114.35(\mathrm{~d}), 119.79(\mathrm{~d}), 123.96(\mathrm{~s})$ $126.55(\mathrm{~s}), 160.69(\mathrm{~s}) . \mathrm{IR} v_{\max }^{\mathrm{KBr}} \mathrm{cm}^{-1}: 3315(\mathrm{NH}), 1676(\mathrm{C}=\mathrm{O})$. MS (FAB) $m / z$ : $224\left(\mathrm{M}^{+}+1\right)$. Anal. Calcd for $\mathrm{C}_{13} \mathrm{H}_{21} \mathrm{NO}_{2}$ : C, 69.92; H, 9.48; N, 6.27. Found: C, 69.94; H, 9.29; N, 6.15

tert-Butyl 4-Phenylpyrrole-2-carboxylate (20) Compound 20 (56 mg, $41 \%)$ was obtained from $13(234 \mathrm{mg}, 0.56 \mathrm{mmol})$ in a manner similar to that described for 17. It was recrystallized from hexane to give a white powder,

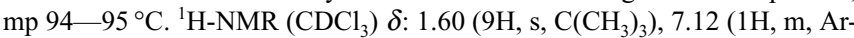
$\mathrm{H}), 7.20(2 \mathrm{H}, \mathrm{s}, 3-\mathrm{H}$ and $5-\mathrm{H}), 7.35(2 \mathrm{H}, \mathrm{t}, J=8.0 \mathrm{~Hz}, \mathrm{Ar}-\mathrm{H}), 7.52(2 \mathrm{H}, \mathrm{d}$ $J=8.0 \mathrm{~Hz}, \mathrm{Ar}-\mathrm{H}), 9.45\left(1 \mathrm{H}\right.$, br s, NH). ${ }^{13} \mathrm{C}-\mathrm{NMR}\left(\mathrm{CDCl}_{3}\right) \delta: 28.41$ (q) 81.14 (s), 111.95 (d), 118.88 (d), 125.13 (s), 125.30 (d), 126.15 (d), 126.61 (s), 128.72 (d), 134.77 (s), 160.79 (s). IR $v_{\max }^{\mathrm{KBr}} \mathrm{cm}^{-1}: 3300$ (NH), 1678 $(\mathrm{C}=\mathrm{O})$. HR-MS (FAB) $\mathrm{m} / \mathrm{z}: 243.1260\left(\right.$ Calcd for $\left.\mathrm{C}_{15} \mathrm{H}_{17} \mathrm{NO}_{2}: 243.1259\right)$.

Methyl (2S)-4-Methylene-1-p-toluenesulfonylpyrrolidine-2-carboxylate (21) $\mathrm{Ph}_{3} \mathrm{P}-\mathrm{CH}_{3} \mathrm{Br}(2.20 \mathrm{~g}, 6.2 \mathrm{mmol})$ was heated at $120^{\circ} \mathrm{C}$ under reduced pressure for $1 \mathrm{~h}$, then heated in a stream of dry $\mathrm{N}_{2}$ at $120^{\circ} \mathrm{C}$. Under an 
argon atmosphere, $\mathrm{Et}_{2} \mathrm{O}(15 \mathrm{ml})$ and $1.9 \mathrm{M} \mathrm{PhLi}(1.8 \mathrm{ml}, 3.4 \mathrm{mmol})$ were added and stirred at room temperature for $4 \mathrm{~h}$. A solution of $5(893 \mathrm{mg}$, $3.00 \mathrm{mmol})$ in THF $(5 \mathrm{ml})$ was added and the mixture was stirred at room temperature for $30 \mathrm{~h}$. The mixture was cooled at $0{ }^{\circ} \mathrm{C}$ and neutralized with $10 \%$ aqueous citric acid. The whole was extracted with AcOEt $(30 \mathrm{ml})$ and water $(20 \mathrm{ml})$. The aqueous layer was extracted with AcOEt $(20 \mathrm{ml} \times 3)$ and the combined organic layer was dried over anhydrous $\mathrm{MgSO}_{4}$, and concentrated under reduced pressure. The residual reddish brown oil was subjected to flash chromatography on silica gel [hexane- $\mathrm{CHCl}_{3}(1: 1)$, then hexane$\left.\mathrm{Et}_{2} \mathrm{O}(3: 1)\right]$ to give $21(91 \mathrm{mg}, 10 \%)$ as a colorless oil. Colorless oil, $[\alpha]_{\mathrm{D}}^{23}$ $-30.8^{\circ}\left(c=0.7, \mathrm{CHCl}_{3}\right) .{ }^{1} \mathrm{H}-\mathrm{NMR}\left(\mathrm{CDCl}_{3}\right) \delta: 2.42\left(3 \mathrm{H}, \mathrm{s}, \mathrm{Ar}-\mathrm{CH}_{3}\right), 2.62$ $\left(1 \mathrm{H}, \mathrm{d}, J=14.2,3.5 \mathrm{~Hz}, 3-\mathrm{H}_{\beta}\right), 2.75\left(1 \mathrm{H}, \mathrm{m}, 3-\mathrm{H}_{\alpha}\right), 3.65\left(3 \mathrm{H}, \mathrm{s}, \mathrm{CO}_{2} \mathrm{CH}_{3}\right)$, $4.02(2 \mathrm{H}, \mathrm{s}, 5-\mathrm{H}), 4.45(1 \mathrm{H}, \mathrm{dd}, J=9.2,3.5 \mathrm{~Hz}, 2-\mathrm{H}), 4.96(2 \mathrm{H}$, s, olefinic $\mathrm{H}), 7.31(2 \mathrm{H}, \mathrm{d}, J=8.4 \mathrm{~Hz}, \mathrm{Ar}-\mathrm{H}), 7.73(2 \mathrm{H}, \mathrm{d}, J=8.4 \mathrm{~Hz}, \mathrm{Ar}-\mathrm{H}) .{ }^{13} \mathrm{C}-\mathrm{NMR}$ $\left(\mathrm{CDCl}_{3}\right) \delta: 21.56(\mathrm{q}), 36.92(\mathrm{t}), 51.75(\mathrm{t}), 52.44(\mathrm{q}), 60.45(\mathrm{~d}), 108.65$ (t), 127.49 (d), 129.72 (d), 134.99 (s), 141.94 (s), 143.85 (s), 171.80 (s). IR $v_{\max }^{\text {neat }} \mathrm{cm}^{-1}: 1741(\mathrm{C}=\mathrm{O})$. HR-MS (FAB) $\mathrm{m} / \mathrm{z}$ : 296.0957 (Calcd for $\left.\mathrm{C}_{14} \mathrm{H}_{18} \mathrm{NO}_{4} \mathrm{~S}: 296.0957\right)$.

tert-Butyl (2S)-4-Methylene-1-p-toluenesulfonylpyrrolidine-2-carboxylate (22) Under an argon atmosphere, $0.5 \mathrm{M}$ Tebbe reagent in toluene $(4.0 \mathrm{ml}, 2.0 \mathrm{mmol})$ was added dropwise to a solution of $6(339 \mathrm{mg}$, $1.00 \mathrm{mmol})$ in THF $(10 \mathrm{ml})$ and the mixture was stirred at $-70^{\circ} \mathrm{C}$ for $4 \mathrm{~h}$, then at $0{ }^{\circ} \mathrm{C}$ for $1 \mathrm{~h}$. Water $(5 \mathrm{ml})$ was added and the insoluble material was filtered out using Hyflo Super-Cel ${ }^{\circledR}$. The filtrate was extracted with AcOEt $(80 \mathrm{ml} \times 2)$. The organic layer was washed with water $(50 \mathrm{ml})$, dried over anhydrous $\mathrm{Na}_{2} \mathrm{SO}_{4}$, and concentrated under reduced pressure. The residual reddish brown oil was subjected to flash chromatography on silica gel [hexane-AcOEt $(6: 1)$, then hexane- $\mathrm{CHCl}_{3}(1: 3)$ ] to give $22(54 \mathrm{mg}, 16 \%)$ as a pale yellow oil and $\mathbf{2 3}(78 \mathrm{mg}, 22 \%)$ as a white solid, which was recrystallized from hexane to give a white powder, $\mathrm{mp} 76-77^{\circ} \mathrm{C}$.

22: $[\alpha]_{\mathrm{D}}^{31}-38.8^{\circ}\left(c=0.4, \mathrm{CHCl}_{3}\right) .{ }^{1} \mathrm{H}-\mathrm{NMR}\left(\mathrm{CDCl}_{3}\right) \delta: 1.41(9 \mathrm{H}, \mathrm{s}$, $\left.\mathrm{C}\left(\mathrm{CH}_{3}\right)_{3}\right), 2.42\left(3 \mathrm{H}, \mathrm{s}, \mathrm{Ar}-\mathrm{CH}_{3}\right), 2.55\left(1 \mathrm{H}, \mathrm{dd}, J=15.8,3.2 \mathrm{~Hz}, 3-\mathrm{H}_{\beta}\right), 2.76$ $\left(1 \mathrm{H}, \mathrm{dd}, J=15.8,9.0 \mathrm{~Hz}, 3-\mathrm{H}_{\alpha}\right), 4.02(2 \mathrm{H}, \mathrm{s}$, olefinic $\mathrm{H}), 4.35(1 \mathrm{H}, \mathrm{dd}$ $J=3.2,9.0 \mathrm{~Hz}, 2-\mathrm{H}), 4.96(2 \mathrm{H}, \mathrm{m}, 5-\mathrm{H}), 7.30(2 \mathrm{H}, \mathrm{d}, J=8.1 \mathrm{~Hz}, \mathrm{Ar}-\mathrm{H}), 7.74$ $(2 \mathrm{H}, \mathrm{d}, J=8.1 \mathrm{~Hz}, \mathrm{Ar}-\mathrm{H}) .{ }^{13} \mathrm{C}-\mathrm{NMR}\left(\mathrm{CDCl}_{3}\right) \delta: 21.53$ (q), 27.83 (q), 37.29 (t), $51.72(\mathrm{t}), 61.22(\mathrm{~d}), 81.96(\mathrm{~s}), 108.20(\mathrm{t}), 127.46$ (d), $129.64(\mathrm{~d}), 135.73$ (s), $142.54(\mathrm{~s}), 143.56(\mathrm{~s}), 170.45(\mathrm{~s}) . \mathrm{IR} v_{\max }^{\text {neat }} \mathrm{cm}^{-1}: 1743(\mathrm{C}=\mathrm{O})$. HR-MS (FAB) $m / z$ : 338.1424 (Calcd for $\mathrm{C}_{17} \mathrm{H}_{23} \mathrm{NO}_{4} \mathrm{~S}: 338.1426$ ).

23: $[\alpha]_{\mathrm{D}}^{25}-85.7^{\circ}\left(c=0.4, \mathrm{CHCl}_{3}\right)$. ${ }^{1} \mathrm{H}-\mathrm{NMR}\left(\mathrm{CDCl}_{3}\right) \delta: 0.75(3 \mathrm{H}, \mathrm{s}$, $\left.\mathrm{CH}_{3}\right), 1.07\left(3 \mathrm{H}, \mathrm{s}, \mathrm{CH}_{3}\right), 1.48\left(9 \mathrm{H}, \mathrm{s}, \mathrm{C}\left(\mathrm{CH}_{3}\right)_{3}\right), 1.76(1 \mathrm{H}, \mathrm{dd}, J=8.2$, $17.6 \mathrm{~Hz}, 3-\mathrm{Ha}), 1.95(1 \mathrm{H}, \mathrm{dd}, J=8.2,17.6 \mathrm{~Hz}, 3-\mathrm{Hb}), 2.42\left(3 \mathrm{H}, \mathrm{s}, \mathrm{Ar}-\mathrm{CH}_{3}\right)$, $3.13(2 \mathrm{H}, \mathrm{m}, 5-\mathrm{H}), 4.17(1 \mathrm{H}, \mathrm{t}, J=8.2 \mathrm{~Hz}, 2-\mathrm{H}), 7.30(2 \mathrm{H}, \mathrm{d}, J=8.1 \mathrm{~Hz}, \mathrm{Ar}-$ $\mathrm{H}), 7.79(2 \mathrm{H}, \mathrm{d}, J=8.1 \mathrm{~Hz}, \mathrm{Ar}-\mathrm{H}) .{ }^{13} \mathrm{C}-\mathrm{NMR}\left(\mathrm{CDCl}_{3}\right) \delta: 21.53(\mathrm{q}), 25.78$ (q), 27.91 (q), $38.74(\mathrm{~s}), 44.57$ (t), 60.52 (t), 61.24 (d), 81.56 (s), $127.62(\mathrm{~d})$, 129.51 (d), 135.64 (s), 143.39 (s), 171.56 (s). IR $v_{\max }^{\mathrm{KBr}} \mathrm{cm}^{-1}: 1745(\mathrm{C}=\mathrm{O})$. MS (FAB) $m / z$ : $354\left(\mathrm{M}^{+}+1\right)$. Anal. Calcd for $\mathrm{C}_{18} \mathrm{H}_{27} \mathrm{NO}_{4} \mathrm{~S}: \mathrm{C}, 61.16 ; \mathrm{H}$, 7.70; N, 3.96. Found: C, 61.14; H, 7.56; N, 4.01 .

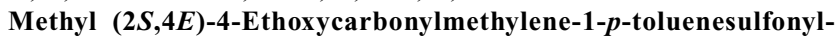
pyrrolidine-2-carboxylate (24a) and Methyl (2S,4Z)-4-Ethoxycarbonylmethylene-1-p-toluenesulfonylpyrrolidine-2-carboxylate (24b) THF (10 $\mathrm{ml}$ ) was added to $60 \% \mathrm{NaH}$ in oil $(168 \mathrm{mg}, 4.2 \mathrm{mmol})$, which was washed beforehand with hexane to remove the oil. Under an argon atmosphere, triethyl phosphonoacetate $(1.12 \mathrm{~g}, 5.00 \mathrm{mmol})$ was added and the whole was stirred at room temperature for $2 \mathrm{~h}$. After the mixture was cooled at $-40{ }^{\circ} \mathrm{C}$, a solution of $5(297 \mathrm{mg}, 1.00 \mathrm{mmol})$ in THF $(5 \mathrm{ml})$ was added dropwise and the whole was stirred for $1 \mathrm{~h}$. A sufficient amount of carbon dioxide was bubbled into the mixture and the mixture was concentrated under reduced pressure. Water $(20 \mathrm{ml})$ was added to the residue at $0{ }^{\circ} \mathrm{C}$ and the whole was extracted with benzene $(20 \mathrm{ml} \times 3)$. The organic layer was washed with brine $(20 \mathrm{ml})$, dried over anhydrous $\mathrm{Na}_{2} \mathrm{SO}_{4}$, and concentrated under reduced pressure. The residual yellow oil was subjected to column chromatography on silica gel [hexane-AcOEt $(4: 1)$ ] to give $\mathbf{2 4 a}(177 \mathrm{mg}, 48 \%)$ as a colorless oil, and then $24 \mathrm{~b}(163 \mathrm{mg}, 44 \%)$ as a colorless oil.

24a: $[\alpha]_{\mathrm{D}}^{29}-6.8^{\circ}\left(c=1.0, \mathrm{CHCl}_{3}\right) .{ }^{1} \mathrm{H}-\mathrm{NMR}\left(\mathrm{CDCl}_{3}\right) \delta: 1.25(3 \mathrm{H}, \mathrm{t}$, $\left.J=7.1 \mathrm{~Hz}, \mathrm{CH}_{2} \mathrm{CH}_{3}\right), 2.43\left(3 \mathrm{H}, \mathrm{s}, \mathrm{Ar}-\mathrm{CH}_{3}\right), 3.13(1 \mathrm{H}, \mathrm{dd}, J=19.0,9.4 \mathrm{~Hz}$, $\left.3-\mathrm{H}_{\alpha}\right), 3.32\left(1 \mathrm{H}\right.$, brd, $\left.J=19.0 \mathrm{~Hz}, 3-\mathrm{H}_{\beta}\right), 3.64\left(3 \mathrm{H}, \mathrm{s}, \mathrm{COOCH}_{3}\right), 4.13(2 \mathrm{H}$, q, $\left.J=7.1 \mathrm{~Hz}, \mathrm{CH}_{2} \mathrm{CH}_{3}\right), 4.22\left(1 \mathrm{H}, \mathrm{d}, J=16.0 \mathrm{~Hz}, 5-\mathrm{H}_{\beta}\right), 4.24(1 \mathrm{H}, \mathrm{d}$, $\left.J=16.0 \mathrm{~Hz}, 5-\mathrm{H}_{\alpha}\right), 4.54(1 \mathrm{H}, \mathrm{dd}, J=9.0,2.9 \mathrm{~Hz}, 2-\mathrm{H}), 5.80(1 \mathrm{H}$, br s, olefinic H), $7.32(2 \mathrm{H}, \mathrm{d}, J=7.9 \mathrm{~Hz}, \mathrm{Ar}-\mathrm{H}), 7.72(2 \mathrm{H}, \mathrm{d}, J=7.9 \mathrm{~Hz}, \mathrm{Ar}-\mathrm{H}) .{ }^{13} \mathrm{C}-\mathrm{NMR}$ $\left(\mathrm{CDCl}_{3}\right) \delta: 14.22(\mathrm{q}), 21.55(\mathrm{q}), 35.89(\mathrm{t}), 52.45(\mathrm{q}), 53.11(\mathrm{t}), 60.24(\mathrm{t})$, 60.57 (d), 113.83 (d), 127.48 (d), 129.84 (d), 134.70 (s), 144.14 (s), 155.42 (s), $165.54(\mathrm{~s}), 171.39(\mathrm{~s})$. IR $v_{\max }^{\text {neat }} \mathrm{cm}^{-1}: 1743,1712(\mathrm{C}=\mathrm{O})$. HR-MS $m / z$ : 367.1093 (Calcd for $\mathrm{C}_{17} \mathrm{H}_{21} \mathrm{NO}_{6} \mathrm{~S}: 367.1090$ ).

24b: $[\alpha]_{\mathrm{D}}^{29}-12.5^{\circ}\left(c=1.1, \mathrm{CHCl}_{3}\right) .{ }^{1} \mathrm{H}-\mathrm{NMR}\left(\mathrm{CDCl}_{3}\right) \delta: 1.27(3 \mathrm{H}, \mathrm{t}$,
$\left.J=7.1 \mathrm{~Hz}, \mathrm{CH}_{2} \mathrm{CH}_{3}\right), 2.43\left(3 \mathrm{H}, \mathrm{s}, \mathrm{Ar}-\mathrm{CH}_{3}\right), 2.82(1 \mathrm{H}$, brd, $J=17.4 \mathrm{~Hz}, 3-$ $\left.\mathrm{H}_{\beta}\right), 2.94\left(1 \mathrm{H}\right.$, ddd, $\left.J=17.4,8.9,1.1 \mathrm{~Hz}, 3-\mathrm{H}_{\alpha}\right), 3.62\left(3 \mathrm{H}, \mathrm{s}, \mathrm{COOC}_{3}\right), 4.15$ $\left(2 \mathrm{H}, \mathrm{q}, J=7.1 \mathrm{~Hz}, \mathrm{CH}_{2} \mathrm{CH}_{3}\right), 4.46-4.58(3 \mathrm{H}, \mathrm{m}, 2-\mathrm{H}$ and $5-\mathrm{H}), 5.78(1 \mathrm{H}, \mathrm{s}$, olefinic H), $7.32(2 \mathrm{H}, \mathrm{d}, J=8.3 \mathrm{~Hz}, \mathrm{Ar}-\mathrm{H}), 7.32(2 \mathrm{H}, \mathrm{d}, J=8.3 \mathrm{~Hz}, \mathrm{Ar}-\mathrm{H})$ ${ }^{13} \mathrm{C}-\mathrm{NMR}\left(\mathrm{CDCl}_{3}\right) \delta: 14.21(\mathrm{q}), 21.56(\mathrm{q}), 37.91(\mathrm{t}), 52.03(\mathrm{t}), 52.47(\mathrm{q})$, 59.03 (d), 60.34 (t), 114.29 (d), 127.52 (d), 129.77 (d), 134.99 (s), 143.96 (s), $155.74(\mathrm{~s}), 165.40(\mathrm{~s}), 171.18(\mathrm{~s})$. IR $v_{\max }^{\text {neat }} \mathrm{cm}^{-1}: 1745,1711(\mathrm{C}=\mathrm{O})$. HR-MS $m / z$ : 367.1092 (Calcd for $\mathrm{C}_{17} \mathrm{H}_{21} \mathrm{NO}_{6} \mathrm{~S}: 367.1090$ ).

tert-Butyl $(2 S, 4 E)-4$-Ethoxycarbonylmethylene-1-p-toluenesulfonylpyrrolidine-2-carboxylate (25a), tert-Butyl $(2 S, 4 Z)-4$-Ethoxycarbonylmethylene-1-p-toluenesulfonylpyrrolidine-2-carboxylate (25b), tert-Butyl (2S)-4-Ethoxycarbonylmethyl-1-p-toluenesulfonyl-3-pyrroline-2-carboxylate (25c), and tert-Butyl (2S)-4-Ethoxycarbonylmethyl-1-p-toluenesulfonyl-4-pyrroline-2-carboxylate (25d) THF (10 ml) was added to $60 \%$ $\mathrm{NaH}$ in oil (168 mg, $4.2 \mathrm{mmol})$, which was washed beforehand with hexane to remove the oil. Under an argon atmosphere, triethyl phosphonoacetate $(1.12 \mathrm{~g}, 5.00 \mathrm{mmol})$ was added and the whole was stirred at room temperature for $2 \mathrm{~h}$. After the mixture was cooled at $0{ }^{\circ} \mathrm{C}$, a solution of $6(339 \mathrm{mg}$, $1.00 \mathrm{mmol})$ in THF $(5 \mathrm{ml})$ was added dropwise and the whole was stirred for $1 \mathrm{~h}$. A sufficient amount of carbon dioxide was bubbled into the mixture and the mixture was concentrated under reduced pressure. Water $(20 \mathrm{ml})$ was added to the residue at $0{ }^{\circ} \mathrm{C}$ and the whole was extracted with benzene $(20 \mathrm{ml} \times 3)$. The organic layer was washed with brine $(20 \mathrm{ml})$, dried over anhydrous $\mathrm{Na}_{2} \mathrm{SO}_{4}$, and concentrated under reduced pressure. The residual yellow oil was subjected to column chromatography on silica gel [hexane- $\mathrm{Et}_{2} \mathrm{O}$ (4: 1)] to give 25a (152 mg, 37\%) as a colorless oil, then $\mathbf{2 5 b}(123 \mathrm{mg}, 30 \%)$ as a colorless oil, then $\mathbf{2 5 d}(9 \mathrm{mg}, 3 \%)$ as a pale yellow oil, and then $\mathbf{2 5 c}$ (124 $\mathrm{mg}, 30 \%)$ as a pale yellow oil.

25a: $[\alpha]_{\mathrm{D}}^{21}-9.2^{\circ}\left(c=0.72, \mathrm{CHCl}_{3}\right) .{ }^{1} \mathrm{H}-\mathrm{NMR}\left(\mathrm{CDCl}_{3}\right) \delta: 1.25(3 \mathrm{H}, \mathrm{t}$, $\left.J=7.1 \mathrm{~Hz}, \mathrm{CH}_{2} \mathrm{CH}_{3}\right), 1.40\left(9 \mathrm{H}, \mathrm{s}, \mathrm{C}\left(\mathrm{CH}_{3}\right)_{3}\right), 2.42\left(3 \mathrm{H}, \mathrm{s}, \mathrm{Ar}-\mathrm{CH}_{3}\right), 3.11(1 \mathrm{H}$, ddd, $\left.J=19.0,9.2,0.9 \mathrm{~Hz}, 3-\mathrm{H}_{\alpha}\right), 3.28\left(1 \mathrm{H}\right.$, br d, $\left.J=19.0 \mathrm{~Hz}, 3-\mathrm{H}_{\beta}\right), 4.14$ $\left(2 \mathrm{H}, \mathrm{q}, J=7.1 \mathrm{~Hz}, \mathrm{CH}_{2} \mathrm{CH}_{3}\right), 4.22(1 \mathrm{H}, \mathrm{d}, J=16.5 \mathrm{~Hz}, 5-\mathrm{Ha}), 4.24(1 \mathrm{H}, \mathrm{d}$, $J=16.5 \mathrm{~Hz}, 5-\mathrm{Hb}), 4.42(1 \mathrm{H}, \mathrm{dd}, J=9.2,3.2 \mathrm{~Hz}, 2-\mathrm{H}), 5.79(1 \mathrm{H}, \mathrm{m}$, olefinic $\mathrm{H}), 7.32(2 \mathrm{H}, \mathrm{d}, J=8.0 \mathrm{~Hz}, \mathrm{Ar}-\mathrm{H}), 7.73(2 \mathrm{H}, \mathrm{d}, J=8.0 \mathrm{~Hz}, \mathrm{Ar}-\mathrm{H}) .{ }^{13} \mathrm{C}-\mathrm{NMR}$ $\left(\mathrm{CDCl}_{3}\right) \delta: 14.23(\mathrm{q}), 21.55(\mathrm{q}), 27.81(\mathrm{q}), 36.06(\mathrm{t}), 53.15(\mathrm{t}), 60.20(\mathrm{t})$, 61.41 (d), 82.31 (s), 113.59 (d), 127.49 (d), 129.76 (d), 135.32 (s), 143.91 (s), $156.05(\mathrm{~s}), 165.65(\mathrm{~s}), 170.18(\mathrm{~s})$. IR $v_{\max }^{\text {neat }} \mathrm{cm}^{-1}: 1736,1712(\mathrm{C}=\mathrm{O})$. HR-MS (FAB) $m / z$ : 410.1634 (Calcd for $\mathrm{C}_{20} \mathrm{H}_{28} \mathrm{NO}_{6} \mathrm{~S}: 410.1637$ ).

25b: $[\alpha]_{\mathrm{D}}^{17}-16.6^{\circ}\left(c=0.70, \mathrm{CHCl}_{3}\right) .{ }^{1} \mathrm{H}-\mathrm{NMR}\left(\mathrm{CDCl}_{3}\right) \delta: 1.25(3 \mathrm{H}, \mathrm{t}$, $\left.J=7.1 \mathrm{~Hz}, \mathrm{CH}_{2} \mathrm{CH}_{3}\right), 1.40\left(9 \mathrm{H}, \mathrm{s}, \mathrm{C}\left(\mathrm{CH}_{3}\right)_{3}\right), 2.42\left(3 \mathrm{H}, \mathrm{s}, \mathrm{Ar}-\mathrm{CH}_{3}\right), 3.12(1 \mathrm{H}$, dd, $\left.J=19.0,9.2 \mathrm{~Hz}, 3-\mathrm{H}_{\beta}\right), 3.28\left(1 \mathrm{H}, \mathrm{d}, J=19.0 \mathrm{~Hz}, 3-\mathrm{H}_{\alpha}\right), 4.13(2 \mathrm{H}, \mathrm{q}$, $\left.J=7.1 \mathrm{~Hz}, \mathrm{CH}_{2} \mathrm{CH}_{3}\right), 4.23(2 \mathrm{H}, \mathrm{d}, J=3.3 \mathrm{~Hz}, 5-\mathrm{H}), 4.42(1 \mathrm{H}, \mathrm{dd}, J=9.2$, $3.3 \mathrm{~Hz}, 2-\mathrm{H}), 5.78(1 \mathrm{H}, \mathrm{s}$, olefinic $\mathrm{H}), 7.30(2 \mathrm{H}, \mathrm{d}, J=8.3 \mathrm{~Hz}, \mathrm{Ar}-\mathrm{H}), 7.73$ $(2 \mathrm{H}, \mathrm{d}, J=8.3 \mathrm{~Hz}, \mathrm{Ar}-\mathrm{H}) .{ }^{13} \mathrm{C}-\mathrm{NMR}\left(\mathrm{CDCl}_{3}\right) \delta: 14.23$ (q), 21.55 (q), 27.81 $(\mathrm{q}), 36.06(\mathrm{t}), 53.15(\mathrm{t}), 60.21(\mathrm{t}), 61.42(\mathrm{~d}), 82.31(\mathrm{~s}), 113.60(\mathrm{~d}), 127.49$ (d), 129.77 (d), 135.31 (s), 143.91 (s), 156.05 (s), 165.65 (s), 170.18 (s). IR $v_{\max }^{\text {neat }} \mathrm{cm}^{-1}: 1736,1712(\mathrm{C}=\mathrm{O})$. HR-MS (FAB) $\mathrm{m} / \mathrm{z}: 410.1635$ (Calcd for $\left.\mathrm{C}_{20} \mathrm{H}_{28} \mathrm{NO}_{6} \mathrm{~S}: 410.1637\right)$.

25c: $[\alpha]_{\mathrm{D}}^{23}-138.7^{\circ}\left(c=0.7, \mathrm{CHCl}_{3}\right) .{ }^{1} \mathrm{H}-\mathrm{NMR}\left(\mathrm{CDCl}_{3}\right) \delta: 1.22(3 \mathrm{H}, \mathrm{t}$, $\left.J=7.2 \mathrm{~Hz}, \mathrm{CH}_{2} \mathrm{CH}_{3}\right), 1.47\left(9 \mathrm{H}, \mathrm{s}, \mathrm{C}\left(\mathrm{CH}_{3}\right)_{3}\right), 2.42\left(3 \mathrm{H}, \mathrm{s}, \mathrm{Ar}-\mathrm{CH}_{3}\right), 3.07(1 \mathrm{H}$ d, $\left.J=16.3 \mathrm{~Hz}, \mathrm{CH}_{2} \mathrm{COOEt}\right), 3.12\left(1 \mathrm{H}, \mathrm{d}, J=16.3 \mathrm{~Hz}, \mathrm{CH}_{2} \mathrm{COOEt}\right), 4.11(2 \mathrm{H}$, q, $\left.J=7.1 \mathrm{~Hz}, \mathrm{CH}_{2} \mathrm{CH}_{3}\right), 4.17(1 \mathrm{H}$, br dd, $J=13.7,5.7 \mathrm{~Hz}, 5-\mathrm{Ha}), 4.22(1 \mathrm{H}, \mathrm{d}$, $J=13.7 \mathrm{~Hz}, 5-\mathrm{Hb}), 4.98(1 \mathrm{H}, \mathrm{d}, J=2.1 \mathrm{~Hz}, 2-\mathrm{H}), 5.53(1 \mathrm{H}, \mathrm{dd}, J=5.7$, $2.1 \mathrm{~Hz}, 3-\mathrm{H}), 7.31(2 \mathrm{H}, \mathrm{d}, J=8.1 \mathrm{~Hz}, \mathrm{Ar}-\mathrm{H}), 7.79(2 \mathrm{H}, \mathrm{d}, J=8.1 \mathrm{~Hz}, \mathrm{Ar}-\mathrm{H})$. ${ }^{13} \mathrm{C}-\mathrm{NMR}\left(\mathrm{CDCl}_{3}\right) \delta: 14.09(\mathrm{q}), 21.55(\mathrm{q}), 27.96(\mathrm{q}), 34.57(\mathrm{t}), 56.54(\mathrm{t})$, 61.15 (t), 68.97 (d), 82.26 (s), 122.23 (d), 127.59 (d), 129.68 (d), 134.80 (s), $135.51(\mathrm{~s}), 143.63(\mathrm{~s}), 168.79(\mathrm{~s}), 169.20(\mathrm{~s}) . \mathrm{IR} v_{\max }^{\text {neat }} \mathrm{cm}^{-1}: 1738(\mathrm{C}=\mathrm{O})$. HR-MS (FAB) $m / z: 410.1639$ (Calcd for $\mathrm{C}_{20} \mathrm{H}_{28} \mathrm{NO}_{6} \mathrm{~S}: 410.1637$ ).

25d: $[\alpha]_{\mathrm{D}}^{23}-120.9^{\circ}\left(c=0.3, \mathrm{CHCl}_{3}\right) .{ }^{1} \mathrm{H}-\mathrm{NMR}\left(\mathrm{CDCl}_{3}\right) \delta$ : $1.22(3 \mathrm{H}, \mathrm{t}$, $\left.J=7.1 \mathrm{~Hz}, \mathrm{CH}_{2} \mathrm{CH}_{3}\right), 1.49\left(9 \mathrm{H}, \mathrm{s}, \mathrm{C}\left(\mathrm{CH}_{3}\right)_{3}\right), 2.43\left(3 \mathrm{H}, \mathrm{s}, \mathrm{Ar}-\mathrm{CH}_{3}\right), 2.62(1 \mathrm{H}$, dd, $\left.J=16.0,7.1 \mathrm{~Hz}, 3-\mathrm{H}_{\beta}\right), 2.79\left(1 \mathrm{H}, \mathrm{dd}, J=16.0,11.5 \mathrm{~Hz}, 3-\mathrm{H}_{\alpha}\right), 3.01(1 \mathrm{H}$, d, $\left.J=16.5 \mathrm{~Hz}, \mathrm{CH}_{2} \mathrm{COOEt}\right), 3.03\left(1 \mathrm{H}, \mathrm{d}, J=16.5 \mathrm{~Hz}, \mathrm{CH}_{2} \mathrm{COOEt}\right), 4.10(2 \mathrm{H}$, q, $\left.J=7.2 \mathrm{~Hz}, \mathrm{CH}_{2} \mathrm{CH}_{3}\right), 4.15(1 \mathrm{H}, \mathrm{dd}, J=11.5,7.1 \mathrm{~Hz}, 2-\mathrm{H}), 6.28(1 \mathrm{H}, \mathrm{s}, 5-$ $\mathrm{H}), 7.31(2 \mathrm{H}, \mathrm{d}, J=8.2 \mathrm{~Hz}, \mathrm{Ar}-\mathrm{H}), 7.70(2 \mathrm{H}, \mathrm{d}, J=8.2 \mathrm{~Hz}, \mathrm{Ar}-\mathrm{H}) .{ }^{13} \mathrm{C}-\mathrm{NMR}$ $\left(\mathrm{CDCl}_{3}\right) \delta: 14.12(\mathrm{q}), 21.59(\mathrm{q}), 27.90(\mathrm{q}), 33.55(\mathrm{t}), 37.61(\mathrm{t}), 60.88(\mathrm{t})$, 61.31 (d), 82.19 (s), 116.56 (s), 127.44 (s), 127.75 (s), 129.74 (d), 133.70 (s), 144.04 (s), $169.84(\mathrm{~s}), 169.92(\mathrm{~s})$. IR $v_{\max }^{\text {neat }} \mathrm{cm}^{-1}: 1738(\mathrm{C}=\mathrm{O})$. HR-MS $\mathrm{m} / z$ : 409.1557 (Calcd for $\mathrm{C}_{20} \mathrm{H}_{27} \mathrm{NO}_{6} \mathrm{~S}: 409.1559$ ).

Methyl (2S,4E)-4-Cyanomethylene-1-p-toluenesulfonylpyrrolidine-2carboxylate (26a) and Methyl (2S,4Z)-4-Cyanomethylene-1-p-toluenesulfonylpyrrolidine-2-carboxylate (26b) Compound $\mathbf{5}(297 \mathrm{mg}, 1.00 \mathrm{mmol})$ was treated in a manner similar to that described for $\mathbf{2 4}$, but using diethyl cyanomethylphosphonate $(886 \mathrm{mg}, 5.00 \mathrm{mmol})$ and column chromatography on silica gel [hexane- $\mathrm{Et}_{2} \mathrm{O}(1: 1)$, giving pyrrole derivative $28(5 \mathrm{mg}, 3 \%)$, 
then 26a (118 mg, 37\%) as a white solid, and then $\mathbf{2 6 b}(107 \mathrm{mg}, 33 \%)$ as a white solid. Compounds 26a and 26b were recrystallized from $i$ - $\mathrm{Pr}_{2} \mathrm{O}$ AcOEt, respectively.

26a: Colorless needles, mp $80-82^{\circ} \mathrm{C},[\alpha]_{\mathrm{D}}^{21}+9.4^{\circ}\left(c=0.8, \mathrm{CHCl}_{3}\right) \cdot{ }^{1} \mathrm{H}-$ $\mathrm{NMR}\left(\mathrm{CDCl}_{3}\right) \delta: 2.44\left(3 \mathrm{H}, \mathrm{s}, \mathrm{Ar}-\mathrm{CH}_{3}\right), 2.98-3.02(2 \mathrm{H}, \mathrm{m}, 3-\mathrm{H}), 3.66(3 \mathrm{H}$ $\left.\mathrm{s}, \mathrm{COOCH}_{3}\right), 4.23(1 \mathrm{H}, \mathrm{d}, J=16.5 \mathrm{~Hz}, 5-\mathrm{Ha}), 4.27(1 \mathrm{H}, \mathrm{d}, J=16.5 \mathrm{~Hz}, 5-\mathrm{Hb})$, $4.60(1 \mathrm{H}, \mathrm{t}, J=6.1 \mathrm{~Hz}, 2-\mathrm{H}), 5.34(1 \mathrm{H}, \mathrm{m}$, olefinic $\mathrm{H}), 7.34(2 \mathrm{H}, \mathrm{d}, J=8.3$ $\mathrm{Hz}, \mathrm{Ar}-\mathrm{H}), 7.71(2 \mathrm{H}, \mathrm{d}, J=8.3 \mathrm{~Hz}, \mathrm{Ar}-\mathrm{H}) .{ }^{13} \mathrm{C}-\mathrm{NMR}\left(\mathrm{CDCl}_{3}\right) \delta: 21.60(\mathrm{q})$, 36.70 (t), 52.14 (q), 52.73 (t), 59.81 (d), 93.73 (d), 115.31 (s), 127.44 (d) $129.95(\mathrm{~d}), 134.62(\mathrm{~s}), 144.50(\mathrm{~s}), 161.30(\mathrm{~s}), 170.62(\mathrm{~s})$. IR $v_{\max }^{\mathrm{KBr}} \mathrm{cm}^{-1}$. $2216(\mathrm{CN}), 1736(\mathrm{C}=\mathrm{O})$. MS $m / z$ : $320\left(\mathrm{M}^{+}\right)$. Anal. Calcd for $\mathrm{C}_{15} \mathrm{H}_{16} \mathrm{~N}_{2} \mathrm{O}_{4} \mathrm{~S}$ : C, 56.24; H, 5.03; N, 8.74. Found: C, 56.10; H, 5.04; N, 8.72.

26b: Colorless needles, mp $102-104^{\circ} \mathrm{C},[\alpha]_{\mathrm{D}}^{21}-21.7^{\circ}\left(c=0.7, \mathrm{CHCl}_{3}\right)$ ${ }^{1} \mathrm{H}-\mathrm{NMR}\left(\mathrm{CDCl}_{3}\right) \delta: 2.43\left(3 \mathrm{H}, \mathrm{s}, \mathrm{Ar}-\mathrm{CH}_{3}\right), 2.82(1 \mathrm{H}, \mathrm{dd}, J=17.6,1.1 \mathrm{~Hz}, 3-$ $\left.\mathrm{H}_{\beta}\right), 2.96\left(1 \mathrm{H}, \mathrm{dd}, J=17.6,8.9 \mathrm{~Hz}, 3-\mathrm{H}_{\alpha}\right), 3.63\left(3 \mathrm{H}, \mathrm{s}, \mathrm{COOCH}_{3}\right), 4.30(1 \mathrm{H}$ d, $\left.J=16.6 \mathrm{~Hz}, 5-\mathrm{H}_{\alpha}\right), 4.36\left(1 \mathrm{H}, \mathrm{d}, J=16.6 \mathrm{~Hz}, 5-\mathrm{H}_{\beta}\right), 4.61(1 \mathrm{H}, \mathrm{dd}, J=8.9$, $3.0 \mathrm{~Hz}, 2-\mathrm{H}), 5.33(1 \mathrm{H}, \mathrm{s}$, olefinic $\mathrm{H}), 7.35(2 \mathrm{H}, \mathrm{d}, J=8.2 \mathrm{~Hz}, \mathrm{Ar}-\mathrm{H})$ $7.73(2 \mathrm{H}, \mathrm{d}, J=8.2 \mathrm{~Hz}, \mathrm{Ar}-\mathrm{H}) .{ }^{13} \mathrm{C}-\mathrm{NMR}\left(\mathrm{CDCl}_{3}\right) \delta: 21.60(\mathrm{q}), 37.25(\mathrm{t})$, $51.83(\mathrm{t}), 52.67(\mathrm{q}), 60.15(\mathrm{~d}), 93.56(\mathrm{~d}), 115.04(\mathrm{~s}), 127.50(\mathrm{~d}), 129.94(\mathrm{~d})$ 134.65 (s), 144.44 (s), 161.53 (s), 170.60 (s). IR $v_{\max }^{\mathrm{KBr}} \mathrm{cm}^{-1}: 2222(\mathrm{CN})$, $1753(\mathrm{C}=\mathrm{O})$. MS m/z: $320\left(\mathrm{M}^{+}\right)$. Anal. Calcd for $\mathrm{C}_{15} \mathrm{H}_{16} \mathrm{~N}_{2} \mathrm{O}_{4} \mathrm{~S}: \mathrm{C}, 56.24$ H, 5.03; N, 8.74. Found: C, 56.22; H, 5.11; N, 8.68.

tert-Butyl $(2 S, 4 E)$-4-Cyanomethylene-1-p-toluenesulfonylpyrrolidine2-carboxylate (27a) and tert-Butyl (2S,4Z)-4-Cyanomethylene-1-p-toluenesulfonylpyrrolidine-2-carboxylate (27b) Compound $\mathbf{6}(339 \mathrm{mg}, 1.00$ $\mathrm{mmol}$ ) was treated in a manner similar to that described for $\mathbf{2 5}$, but using diethyl cyanomethylphosphonate $(886 \mathrm{mg}, 5.00 \mathrm{mmol})$ and column chromatography on silica gel [hexane-Et $\mathrm{O}_{2} \mathrm{O}(2: 1)$ ], giving pyrrole derivative 29 $(4 \mathrm{mg}, 5 \%)$, then $27 \mathbf{a}(174 \mathrm{mg}, 48 \%)$ as a white solid, and then $27 \mathbf{b}(131 \mathrm{mg}$, $36 \%$ ) as a white solid. Compounds $27 \mathbf{a}$ and $27 \mathbf{b}$ were recrystallized from hexane-AcOEt, respectively.

27a: Colorless needles, mp $96-97^{\circ} \mathrm{C},[\alpha]_{\mathrm{D}}^{17}+9.9^{\circ}\left(c=0.7, \mathrm{CHCl}_{3}\right) .{ }^{1} \mathrm{H}-$ NMR $\left(\mathrm{CDCl}_{3}\right) \delta: 1.41\left(9 \mathrm{H}, \mathrm{s}, \mathrm{C}\left(\mathrm{CH}_{3}\right)_{3}\right), 2.44\left(3 \mathrm{H}, \mathrm{s}, \mathrm{Ar}-\mathrm{CH}_{3}\right), 2.94(1 \mathrm{H}$ br d, $\left.J=17.9 \mathrm{~Hz}, 3-\mathrm{H}_{\beta}\right), 2.98\left(1 \mathrm{H}\right.$, ddd, $\left.J=17.9,8.5,1.6 \mathrm{~Hz}, 3-\mathrm{H}_{\alpha}\right), 4.24(1 \mathrm{H}$, dd, $J=16.5,1.1 \mathrm{~Hz}, 5-\mathrm{Ha}), 4.26(1 \mathrm{H}, \mathrm{dd}, J=16.5,1.8 \mathrm{~Hz}, 5-\mathrm{Hb}), 4.49(1 \mathrm{H}$ dd, $J=8.5,3.2 \mathrm{~Hz}, 2-\mathrm{H}), 5.32(1 \mathrm{H}, \mathrm{m}$, olefinic $\mathrm{H}), 7.32(2 \mathrm{H}, \mathrm{d}, J=8.1 \mathrm{~Hz}$, $\mathrm{Ar}-\mathrm{H}), 7.72(2 \mathrm{H}, \mathrm{d}, J=8.1 \mathrm{~Hz}, \mathrm{Ar}-\mathrm{H}) .{ }^{13} \mathrm{C}-\mathrm{NMR}\left(\mathrm{CDCl}_{3}\right) \delta: 21.58(\mathrm{q}), 27.80$ (q), $37.03(\mathrm{t}), 52.15(\mathrm{t}), 60.70(\mathrm{~d}), 82.95$ (s), 93.46 (d), 115.37 (s), 127.44 (d), 129.90 (d), $135.16(\mathrm{~s}), 144.29$ (s), 161.90 (s), 169.36 (s). IR $v_{\max }^{\text {neat }} \mathrm{cm}^{-1}$ $2222(\mathrm{CN}), 1739(\mathrm{C}=\mathrm{O})$. MS (FAB) $m / z: 363\left(\mathrm{M}^{+}+1\right)$. Anal. Calcd for $\mathrm{C}_{18} \mathrm{H}_{22} \mathrm{~N}_{2} \mathrm{O}_{4} \mathrm{~S}: \mathrm{C}, 59.65 ; \mathrm{H}, 6.12 ; \mathrm{N}, 7.73$. Found: C, 59.56; H, 6.18; N, 7.77.

27b: Colorless needles, mp $120-121^{\circ} \mathrm{C},[\alpha]_{\mathrm{D}}^{17}-37.2^{\circ}\left(c=0.6, \mathrm{CHCl}_{3}\right)$ ${ }^{1} \mathrm{H}-\mathrm{NMR}\left(\mathrm{CDCl}_{3}\right) \delta: 1.38\left(9 \mathrm{H}, \mathrm{s}, \mathrm{C}\left(\mathrm{CH}_{3}\right)_{3}\right), 2.44\left(3 \mathrm{H}, \mathrm{s}, \mathrm{Ar}-\mathrm{CH}_{3}\right), 2.75(1 \mathrm{H}$, br d, $\left.J=17.6 \mathrm{~Hz}, 3-\mathrm{H}_{\beta}\right), 2.96\left(1 \mathrm{H}, \mathrm{dd}, J=17.6,9.0 \mathrm{~Hz}, 3-\mathrm{H}_{\alpha}\right), 4.34(1 \mathrm{H}, \mathrm{brd}$, $\left.J=16.6 \mathrm{~Hz}, 5-\mathrm{H}_{\beta}\right), 4.37\left(1 \mathrm{H}, \mathrm{dd}, J=16.6,1.1 \mathrm{~Hz}, 5-\mathrm{H}_{\alpha}\right), 4.49(1 \mathrm{H}$, ddd, $J=9.0,2.5,1.6 \mathrm{~Hz}, 2-\mathrm{H}), 5.33(1 \mathrm{H}, \mathrm{m}$, olefinic $\mathrm{H}), 7.33(2 \mathrm{H}, \mathrm{d}, J=8.1 \mathrm{~Hz}$, $\mathrm{Ar}-\mathrm{H}), 7.74(2 \mathrm{H}, \mathrm{d}, J=8.1 \mathrm{~Hz}, \mathrm{Ar}-\mathrm{H}) .{ }^{13} \mathrm{C}-\mathrm{NMR}\left(\mathrm{CDCl}_{3}\right) \delta: 21.56(\mathrm{q}), 27.76$ (q), 37.59 (t), $51.85(\mathrm{t}), 61.08$ (d), 82.88 (s), 93.19 (d), 115.07 (s), 127.47 (d), 129.90 (d), 135.22 (s), 144.23 (s), $162.21(\mathrm{~s}), 169.32(\mathrm{~s}) . \mathrm{IR} v_{\max }^{\mathrm{KBr}} \mathrm{cm}^{-1}$ : $2220(\mathrm{CN}), 1743(\mathrm{C}=\mathrm{O})$. MS (FAB) $m / z: 363\left(\mathrm{M}^{+}+1\right)$. Anal. Calcd for $\mathrm{C}_{18} \mathrm{H}_{22} \mathrm{~N}_{2} \mathrm{O}_{4} \mathrm{~S}$ : C, 59.65; H, 6.12; N, 7.73. Found: C, 59.65; H, 6.14; N, 7.72.

Treatments of 21 with Bases Method a: DBU $(0.21 \mathrm{ml}, 1.4 \mathrm{mmol})$ was added to a solution of $21(59 \mathrm{mg}, 0.20 \mathrm{mmol})$ in toluene $(1 \mathrm{ml})$ and the mixture was refluxed for $12 \mathrm{~h}$. After cooling, the mixture was concentrated under reduced pressure and the residual brown oil was subjected to column chromatography on silica gel (AcOEt) to give 4 (27 mg, 97\%).

Method b: To a solution of $21(86 \mathrm{mg}, 0.29 \mathrm{mmol})$ in THF $(2 \mathrm{ml}), 1 \mathrm{~m} t$ $\mathrm{BuOK}$ in THF $(0.7 \mathrm{ml})$ was added and the resulting solution was stirred at room temperature for $1 \mathrm{~h}$. A sufficient amount of carbon dioxide was bubbled into the mixture and the mixture was concentrated under reduced pressure. Water $(15 \mathrm{ml})$ was added to the residue at $0{ }^{\circ} \mathrm{C}$ and the whole was extracted with $\mathrm{CHCl}_{3}(15 \mathrm{ml} \times 2)$. The organic layer was washed with water $(5 \mathrm{ml})$, dried over anhydrous $\mathrm{Na}_{2} \mathrm{SO}_{4}$, and concentrated under reduced pressure. The residue was subjected to column chromatography on silica gel [hexane-AcOEt $(3: 1)$ ] to give 4 (31 $\mathrm{mg}, 77 \%)$.

Treatments of 22 with Bases Method a: DBU $(0.21 \mathrm{ml}, 1.4 \mathrm{mmol})$ was added to a solution of $22(68 \mathrm{mg}, 0.20 \mathrm{mmol})$ in toluene $(1 \mathrm{ml})$ and the mixture was refluxed for $12 \mathrm{~h}$. After cooling, the mixture was concentrated under reduced pressure and the residual brown oil was subjected to column chromatography on silica gel [hexane-AcOEt (3:1)] to give 17 (36 mg, $99 \%$ ).

Method b: To a solution of $22(96 \mathrm{mg}, 0.29 \mathrm{mmol})$ in THF $(2 \mathrm{ml}), 1 \mathrm{M} t$ $\mathrm{BuOK}$ in THF $(0.7 \mathrm{ml})$ was added and the resulting solution was stirred at room temperature for $1 \mathrm{~h}$. A sufficient amount of carbon dioxide was bub- bled into the mixture and the mixture was concentrated under reduced pressure. Water $(15 \mathrm{ml})$ was added to the residue at $0{ }^{\circ} \mathrm{C}$ and the whole was extracted with $\mathrm{CHCl}_{3}(15 \mathrm{ml} \times 2)$. The organic layer was washed with water $(5 \mathrm{ml})$, dried over anhydrous $\mathrm{Na}_{2} \mathrm{SO}_{4}$, and concentrated under reduced pressure. The residue was subjected to column chromatography on silica gel [hexane-AcOEt (3: 1)] to give 17 (44 mg, 85\%).

Methyl 4-(Ethoxycarbonylmethyl)pyrrole-2-carboxylate (28) DBU $(0.21 \mathrm{ml}, 1.4 \mathrm{mmol})$ was added to a solution of $\mathbf{2 4 a}(72 \mathrm{mg}, 0.20 \mathrm{mmol})$ in toluene $(1 \mathrm{ml})$ and the mixture was stirred at room temperature for $1 \mathrm{~h}$. It was concentrated under reduced pressure and the residual brown oil was subjected to column chromatography on silica gel (AcOEt) to give $\mathbf{2 8}$ $(40 \mathrm{mg}, 97 \%)$ as a white solid. It was recrystallized from hexane-AcOEt to give colorless needles, mp $66-68^{\circ} \mathrm{C}$, (lit. ${ }^{27)} \mathrm{mp} 64-65^{\circ} \mathrm{C}$ ). ${ }^{1} \mathrm{H}-\mathrm{NMR}$ $\left(\mathrm{CDCl}_{3}\right) \delta: 1.26\left(3 \mathrm{H}, \mathrm{t}, J=7.1 \mathrm{~Hz}, \mathrm{CH}_{2} \mathrm{CH}_{3}\right), 3.49\left(2 \mathrm{H}, \mathrm{s}, \mathrm{CH}_{2} \mathrm{CO}_{2} \mathrm{Et}\right), 3.84$ $\left(3 \mathrm{H}, \mathrm{s}, \mathrm{CO}_{2} \mathrm{CH}_{3}\right), 4.16\left(2 \mathrm{H}, \mathrm{q}, J=7.1 \mathrm{~Hz}, \mathrm{CH}_{2} \mathrm{CH}_{3}\right), 6.85(1 \mathrm{H}, \mathrm{s}, 3-\mathrm{H}), 6.91$ $(1 \mathrm{H}, \mathrm{s}, 5-\mathrm{H}), 9.41(1 \mathrm{H}, \mathrm{s}, \mathrm{NH}) .{ }^{13} \mathrm{C}-\mathrm{NMR}\left(\mathrm{CDCl}_{3}\right) \delta: 14.21(\mathrm{q}), 32.82(\mathrm{t})$, 51.45 (q), 60.83 (t), 115.73 (d), 117.74 (s), 122.13 (d), 122.54 (s), 161.63 (s), 171.91 (s). IR $v_{\max }^{\mathrm{KBr}} \mathrm{cm}^{-1}: 3325(\mathrm{NH}), 1736,1689(\mathrm{C}=\mathrm{O})$. MS $m / z: 211$ $\left(\mathrm{M}^{+}\right)$. Anal. Calcd for $\mathrm{C}_{10} \mathrm{H}_{13} \mathrm{NO}_{4}: \mathrm{C}, 56.86 ; \mathrm{H}, 6.20 ; \mathrm{N}, 6.63$. Found: $\mathrm{C}$, 56.87; H, 6.15; N, 6.59 .

tert-Butyl 4-(Ethoxycarbonylmethyl)pyrrole-2-carboxylate (29) DBU $(0.21 \mathrm{ml}, 1.4 \mathrm{mmol})$ was added to a solution of $25 \mathrm{a}(82 \mathrm{mg}, 0.20 \mathrm{mmol})$ in toluene $(1 \mathrm{ml})$ and the mixture was refluxed for $1 \mathrm{~h}$. After cooling, the mixture was concentrated under reduced pressure and the residual brown oil was subjected to column chromatography on silica gel [benzene- $\mathrm{Et}_{2} \mathrm{O}(5: 1)$ ] to give $29(50 \mathrm{mg}, 99 \%)$ as a colorless oil. ${ }^{1} \mathrm{H}-\mathrm{NMR}\left(\mathrm{CDCl}_{3}\right) \delta: 1.27(3 \mathrm{H}, \mathrm{t}$, $\left.J=7.1 \mathrm{~Hz}, \mathrm{CH}_{2} \mathrm{CH}_{3}\right), 1.55\left(9 \mathrm{H}, \mathrm{s}, \mathrm{C}\left(\mathrm{CH}_{3}\right)_{3}\right), 3.48(2 \mathrm{H}, \mathrm{s}$, benzylic $\mathrm{H}), 4.16$ $\left(2 \mathrm{H}, \mathrm{q}, J=7.1 \mathrm{~Hz}, \mathrm{CO}_{2} \mathrm{CH}_{2} \mathrm{CH}_{3}\right), 6.77(1 \mathrm{H}, \mathrm{s}, 3-\mathrm{H}), 6.86(1 \mathrm{H}, \mathrm{s}, 5-\mathrm{H}), 9.29$ $\left(1 \mathrm{H}\right.$, br s, NH). ${ }^{13} \mathrm{C}-\mathrm{NMR}\left(\mathrm{CDCl}_{3}\right) \delta: 14.21(\mathrm{q}), 28.37(\mathrm{q}), 32.84(\mathrm{t}), 60.79$ (t), 80.85 (s), 115.03 (d), 117.44 (s), 121.24 (d), 124.35 (s), 160.71 (s), $172.00(\mathrm{~s})$. IR $v_{\max }^{\text {neat }} \mathrm{cm}^{-1}: 3311(\mathrm{NH}), 1738,1678(\mathrm{C}=\mathrm{O}) . \mathrm{HR}-\mathrm{MS} \mathrm{m} / \mathrm{z}$ : 253.1315 (Calcd for $\mathrm{C}_{13} \mathrm{H}_{19} \mathrm{NO}_{4} \mathrm{~S}: 253.1314$ ).

Methyl 4-Cyanomethylpyrrole-2-carboxylate (30) DBU $(0.21 \mathrm{ml}, 1.4$ $\mathrm{mmol})$ was added to a solution of $\mathbf{2 6 a}(65 \mathrm{mg}, 0.20 \mathrm{mmol})$ in toluene $(1 \mathrm{ml})$ and the mixture was stirred at room temperature for $1 \mathrm{~h}$. It was concentrated under reduced pressure and the residual brown oil was subjected to column chromatography on silica gel [hexane-Et $\mathrm{O}(4: 1)$ ] to give 30 (33 mg, 99\%) as a white solid. It was recrystallized from hexane-AcOEt to give colorless prisms, mp 92-93 ${ }^{\circ} \mathrm{C} .{ }^{1} \mathrm{H}-\mathrm{NMR}\left(\mathrm{CDCl}_{3}\right) \delta: 3.60\left(2 \mathrm{H}, \mathrm{s}, \mathrm{CH}_{2} \mathrm{CN}\right), 3.86(3 \mathrm{H}$, $\left.\mathrm{s}, \mathrm{CO}_{2} \mathrm{CH}_{3}\right), 6.85(1 \mathrm{H}, \mathrm{s}, 3-\mathrm{H}), 6.70(1 \mathrm{H}, \mathrm{s}, 5-\mathrm{H}), 9.74(1 \mathrm{H}, \mathrm{s}, \mathrm{NH}) .{ }^{13} \mathrm{C}-$ $\operatorname{NMR}\left(\mathrm{CDCl}_{3}\right) \delta: 15.76(\mathrm{t}), 51.75(\mathrm{q}), 113.95$ (s), 114.48 (d), 118.25 (s), 121.51 (d), 123.34 (s), 161.47 (s). IR $v_{\max }^{\mathrm{KBr}} \mathrm{cm}^{-1}: 3303(\mathrm{NH}), 2249(\mathrm{CN})$ $1685(\mathrm{C}=\mathrm{O})$. MS m/z: $164\left(\mathrm{M}^{+}\right)$. Anal. Calcd for $\mathrm{C}_{8} \mathrm{H}_{8} \mathrm{~N}_{2} \mathrm{O}_{2}: \mathrm{C}, 58.53 ; \mathrm{H}$, $4.91 ; \mathrm{N}, 17.06$. Found: C, 58.50; H, 5.02; N, 17.04 .

tert-Butyl 4-Cyanomethylpyrrole-2-carboxylate (31) DBU $(0.21 \mathrm{ml}$, $1.4 \mathrm{mmol})$ was added to a solution of $27 \mathbf{a}(73 \mathrm{mg}, 0.20 \mathrm{mmol})$ in toluene $(1 \mathrm{ml})$ and the mixture was refluxed for $1 \mathrm{~h}$. After cooling, the mixture was concentrated under reduced pressure and the residual brown oil was subjected to column chromatography on silica gel [benzene- $\mathrm{Et}_{2} \mathrm{O}(5: 1)$ ] to give $31(41 \mathrm{mg}, 99 \%)$ as a white solid. It was recrystallized from hexane-AcOEt to give colorless needles, mp $112-113^{\circ} \mathrm{C} .{ }^{1} \mathrm{H}-\mathrm{NMR}\left(\mathrm{CDCl}_{3}\right) \delta: 1.57(9 \mathrm{H}$, $\left.\mathrm{s}, \mathrm{C}\left(\mathrm{CH}_{3}\right)_{3}\right), 3.59\left(2 \mathrm{H}, \mathrm{s}, \mathrm{CH}_{2} \mathrm{CN}\right), 6.78(1 \mathrm{H}, \mathrm{s}, 3-\mathrm{H}), 6.90(1 \mathrm{H}, \mathrm{s}, 5-\mathrm{H}), 9.61$ $(1 \mathrm{H}, \mathrm{s}, \mathrm{NH}) .{ }^{13} \mathrm{C}-\mathrm{NMR}\left(\mathrm{CDCl}_{3}\right) \delta: 15.80(\mathrm{t}), 28.34(\mathrm{q}), 81.45(\mathrm{~s}), 113.64(\mathrm{~s})$, $113.89(\mathrm{~d}), 118.28(\mathrm{~s}), 120.63(\mathrm{~d}), 125.16(\mathrm{~s}), 160.50$ (s). IR $v_{\max }^{\mathrm{KBr}} \mathrm{cm}^{-1}$ : $3300(\mathrm{NH}), 2252(\mathrm{CN}), 1662(\mathrm{C}=\mathrm{O})$. MS (FAB) m/z: $207\left(\mathrm{M}^{+}+1\right)$. Anal. Calcd for $\mathrm{C}_{11} \mathrm{H}_{14} \mathrm{~N}_{2} \mathrm{O}_{2}$ : C, 64.06; H, 6.84; N, 13.58. Found: C, 64.20; H, 6.85; N, 13.67.

tert-Butyl (2S,4R)-4-Ethoxycarbonylmethyl-4-hydroxy-1-p-toluenesulfonylpyrrolidine-2-carboxylate (32a) and tert-Butyl $(2 S, 4 S)-4$-Ethoxycarbonylmethyl-4-hydroxy-1-p-toluenesulfonylpyrrolidine-2-carboxylate (32b) A mixture of granulated zinc $(0.15 \mathrm{~g})$, copper(II) acetate hydrate $(15 \mathrm{mg})$, and acetic acid $(1 \mathrm{ml})$ was stirred at room temperature for $12 \mathrm{~h}$. The acetic acid was then decanted and the $\mathrm{Zn}-\mathrm{Cu}$ couple was washed with $\mathrm{Et}_{2} \mathrm{O}$ $(1 \mathrm{ml} \times 3)$ and benzene $(1 \mathrm{ml})$. To the $\mathrm{Zn}-\mathrm{Cu}$ couple, THF $(2 \mathrm{ml})$ was added with stirring, then a solution of compound $6(339 \mathrm{mg}, 1.00 \mathrm{mmol})$ and ethyl bromoacetate $(334 \mathrm{mg}, 2.0 \mathrm{mmol})$ in THF $(2 \mathrm{ml})$ was added dropwise. The mixture was heated under reflux for $1 \mathrm{~h}$. After cooling, AcOEt $(5 \mathrm{ml})$ and concentrated ammonium hydroxide $(5 \mathrm{ml})$ were added at $0{ }^{\circ} \mathrm{C}$ and the mixture was stirred for $10 \mathrm{~min}$. The whole was filtered and the organic layer was separated. The aqueous layer was extracted with AcOEt $(5 \mathrm{ml} \times 2)$ and the combined organic layer was washed with water $(10 \mathrm{ml})$, then brine $(10 \mathrm{ml})$. It was dried over anhydrous $\mathrm{Na}_{2} \mathrm{SO}_{4}$, and concentrated under reduced pressure. The residual yellow oil was subjected to flash chromatography on silica gel [hexane-AcOEt $(4: 1)$ ], then column chromatography on silica gel [ben- 
zene-AcOEt (10:1)], giving 32a (192 mg, 45\%) as a white solid and 32b (43 mg, 10\%) as a colorless oil. Compound 32a was recrystallized from hexane-AcOEt.

32a: Colorless prisms (hexane-AcOEt) : mp $73-74^{\circ} \mathrm{C},[\alpha]_{\mathrm{D}}^{17}-37.4^{\circ}$ $\left(c=0.93, \mathrm{CHCl}_{3}\right) .{ }^{1} \mathrm{H}-\mathrm{NMR}\left(\mathrm{CDCl}_{3}\right) \delta: 1.25\left(3 \mathrm{H}, \mathrm{t}, J=7.1 \mathrm{~Hz}, \mathrm{CH}_{2} \mathrm{CH}_{3}\right)$ $1.48\left(9 \mathrm{H}, \mathrm{s}, \mathrm{C}\left(\mathrm{CH}_{3}\right)_{3}\right), 2.16(2 \mathrm{H}, \mathrm{m}, 3-\mathrm{H}), 2.43\left(3 \mathrm{H}, \mathrm{s}, \mathrm{Ar}-\mathrm{CH}_{3}\right), 2.52(1 \mathrm{H}, \mathrm{d}$, $\left.J=15.4 \mathrm{~Hz}, \mathrm{CH}_{2} \mathrm{CO}_{2} \mathrm{Et}\right), 2.56\left(1 \mathrm{H}, \mathrm{d}, J=15.4 \mathrm{~Hz}, \mathrm{CH}_{2} \mathrm{CO}_{2} \mathrm{Et}\right), 3.28(1 \mathrm{H}, \mathrm{d}$, $\left.J=9.9 \mathrm{~Hz}, 5-\mathrm{H}_{\alpha}\right), 3.58\left(1 \mathrm{H}, \mathrm{dd}, J=9.9,0.8 \mathrm{~Hz}, 5-\mathrm{H}_{\beta}\right), 4.14(2 \mathrm{H}, \mathrm{q}, J=7.1 \mathrm{~Hz}$, $\left.\mathrm{C}_{2} \mathrm{CH}_{3}\right), 4.27(1 \mathrm{H}, \mathrm{dd}, J=8.9,3.2 \mathrm{~Hz}, 2-\mathrm{H}), 4.34(1 \mathrm{H}, \mathrm{s}, \mathrm{OH}), 7.32(2 \mathrm{H}, \mathrm{d}$, $J=8.3 \mathrm{~Hz}, \mathrm{Ar}-\mathrm{H}), 7.77(2 \mathrm{H}, \mathrm{d}, J=8.3 \mathrm{~Hz}, \mathrm{Ar}-\mathrm{H}) .{ }^{13} \mathrm{C}-\mathrm{NMR}\left(\mathrm{CDCl}_{3}\right) \delta: 14.12$ (q), $21.56(\mathrm{q}), 27.83(\mathrm{q}), 41.93(\mathrm{t}), 42.40(\mathrm{t}), 59.44(\mathrm{t}), 59.98(\mathrm{~d}), 60.95(\mathrm{t})$, 76.31 (s), 82.96 (s), 127.68 (d), 129.74 (d), 135.01 (s), 143.87 (s), 170.61 (s), $172.36(\mathrm{~s}) . \mathrm{IR} v_{\max }^{\mathrm{KBr}} \mathrm{cm}^{-1}: 3438(\mathrm{OH}), 1736,1714(\mathrm{C}=\mathrm{O})$. MS (FAB) $m / z$ : $428\left(\mathrm{M}^{+}+1\right)$. Anal. Calcd for $\mathrm{C}_{20} \mathrm{H}_{29} \mathrm{NO}_{7} \mathrm{~S}: \mathrm{C}, 56.19 ; \mathrm{H}, 6.84 ; \mathrm{N}, 3.28$. Found: C, 56.20; H, 6.75; N, 3.21.

32b: $[\alpha]_{\mathrm{D}}^{26}-54.6^{\circ}\left(c=0.40, \mathrm{CHCl}_{3}\right) .{ }^{1} \mathrm{H}-\mathrm{NMR}\left(\mathrm{CDCl}_{3}\right) \delta: 1.26(3 \mathrm{H}, \mathrm{t}$ $\left.J=7.2 \mathrm{~Hz}, \mathrm{CH}_{2} \mathrm{CH}_{3}\right), 1.50\left(9 \mathrm{H}, \mathrm{s}, \mathrm{C}\left(\mathrm{CH}_{3}\right)_{3}\right), 1.90(1 \mathrm{H}, \mathrm{dd}, J=13.0,9.0 \mathrm{~Hz}, 3$ $\left.\mathrm{H}_{\beta}\right), 2.31\left(1 \mathrm{H}\right.$, ddd, $\left.J=13.0,7.7,1.8 \mathrm{~Hz}, 3-\mathrm{H}_{\alpha}\right), 2.42\left(3 \mathrm{H}, \mathrm{s}, \mathrm{Ar}-\mathrm{CH}_{3}\right), 2.64$ $\left(1 \mathrm{H}, \mathrm{d}, J=16.9 \mathrm{~Hz}, \mathrm{CH}_{2} \mathrm{CO}_{2} \mathrm{Et}\right), 2.65\left(1 \mathrm{H}, \mathrm{d}, J=16.9 \mathrm{~Hz}, \mathrm{CH}_{2} \mathrm{CO}_{2} \mathrm{Et}\right), 3.27$ $(1 \mathrm{H}, \mathrm{s}, \mathrm{OH}), 3.41\left(1 \mathrm{H}, \mathrm{d}, J=11.5 \mathrm{~Hz}, 5-\mathrm{H}_{\beta}\right), 3.52(1 \mathrm{H}, \mathrm{dd}, J=11.5,1.8 \mathrm{~Hz}$, $\left.5-\mathrm{H}_{\alpha}\right), 4.15\left(2 \mathrm{H}, \mathrm{q}, J=7.2 \mathrm{~Hz}, \mathrm{CH}_{2} \mathrm{CH}_{3}\right), 4.21(1 \mathrm{H}, \mathrm{dd}, J=9.0,7.7 \mathrm{~Hz}, 2-\mathrm{H})$, $7.32(2 \mathrm{H}, \mathrm{d}, J=8.1 \mathrm{~Hz}, \mathrm{Ar}-\mathrm{H}), 7.78(2 \mathrm{H}, \mathrm{d}, J=8.1 \mathrm{~Hz}, \mathrm{Ar}-\mathrm{H}) .{ }^{13} \mathrm{C}-\mathrm{NMR}$ $\left(\mathrm{CDCl}_{3}\right) \delta: 14.09(\mathrm{q}), 21.57(\mathrm{q}), 27.92(\mathrm{q}), 41.30(\mathrm{t}), 42.98(\mathrm{t}), 59.59(\mathrm{t})$, 60.79 (d), 61.25 (t), 79.14 (s), 81.96 (s), 127.88 (d), 129.55 (d), 134.63 (s), $143.66(\mathrm{~s}), 171.02(\mathrm{~s}), 171.98(\mathrm{~s})$. IR $v_{\max }^{\text {neat }} \mathrm{cm}^{-1}: 3506(\mathrm{OH}), 1738,1732$ $(\mathrm{C}=\mathrm{O})$. HR-MS (FAB) $m / z$ : 428.1744 (Calcd for $\mathrm{C}_{20} \mathrm{H}_{30} \mathrm{NO}_{7} \mathrm{~S}: 428.1743$ ).

An Epimeric Mixture of tert-Butyl $(2 S, 4 R)-4-[1-($ Ethoxycarbonyl)ethyl]-4-hydroxy-1-p-toluenesulfonylpyrrolidine-2-carboxylates (33a) and an Epimeric Mixture of tert-Butyl $(2 S, 4 S)-4-[1-($ Ethoxycarbonyl) ethyl]-4-hydroxy-1-p-toluenesulfonylpyrrolidine-2-carboxylates (33b) Under an argon atmosphere, ethyl 2-bromopropionate $(905 \mathrm{mg}, 5.0 \mathrm{mmol})$ was added to a mixture of $6(679 \mathrm{mg}, 2.00 \mathrm{mmol})$, zinc powder $(260 \mathrm{mg}$, $4.00 \mathrm{mg}$-atoms $)$, trimethyl borate $(1.04 \mathrm{~g}, 10.0 \mathrm{mmol})$, and THF $(10 \mathrm{ml})$. The whole was stirred for a while at room temperature and heated under reflux for $1.5 \mathrm{~h}$. After cooling, AcOEt $(10 \mathrm{ml})$, concentrated ammonium hydroxide $(10 \mathrm{ml})$, and glycerin $(10 \mathrm{ml})$ were added at $0{ }^{\circ} \mathrm{C}$ and the mixture was stirred for $10 \mathrm{~min}$. The whole was filtered and the organic layer was separated. The aqueous layer was extracted with AcOEt $(10 \mathrm{ml} \times 2)$ and the combined organic layer was washed with water $(20 \mathrm{ml})$, then brine $(20 \mathrm{ml})$. It was dried over anhydrous $\mathrm{Na}_{2} \mathrm{SO}_{4}$, and concentrated under reduced pressure. The residual brown oil was subjected to column chromatography on silica gel [hexane-AcOEt $(3: 1)$ ] to give 33a $(486 \mathrm{mg}, 55 \%)$ as a pale yellow oil and 33b $(186 \mathrm{mg}, 21 \%)$ as a pale yellow oil.

tert-Butyl (2S,4R)-4-[2-(Ethoxycarbonyl)propan-2-yl]-4-hydroxy-1-ptoluenesulfonylpyrrolidene-2-carboxylate (34a) and tert-Butyl $(2 S, 4 S)-4$ [2-(Ethoxycarbonyl)propan-2-yl]-4-hydroxy-1-p-toluenesulfonylpyrrolidene-2-carboxylate (34b) Under an argon atmosphere, ethyl 2-bromoisobutyrate $(975 \mathrm{mg}, 5.0 \mathrm{mmol})$ was added to a mixture of $6(679 \mathrm{mg}$, $2.00 \mathrm{mmol})$, zinc powder $(260 \mathrm{mg}, 4.00 \mathrm{mg}$-atoms $)$, trimethyl borate $(1.04 \mathrm{~g}$, $10.0 \mathrm{mmol})$, and THF $(10 \mathrm{ml})$. The whole was stirred for a while at room temperature and heated under reflux for $1 \mathrm{~h}$. After cooling, AcOEt $(10 \mathrm{ml})$, concentrated ammonium hydroxide $(10 \mathrm{ml})$, and glycerin $(10 \mathrm{ml})$ were added at $0{ }^{\circ} \mathrm{C}$ and the mixture was stirred for $10 \mathrm{~min}$. The whole was filtered and the organic layer was separated. The aqueous layer was extracted with AcOEt $(10 \mathrm{ml} \times 2)$ and the combined organic layer was washed with water $(20 \mathrm{ml})$, then brine $(20 \mathrm{ml})$. It was dried over anhydrous $\mathrm{Na}_{2} \mathrm{SO}_{4}$, and concentrated under reduced pressure. The residual brown oil was subjected to flash chromatography on silica gel [hexane- $\mathrm{Et}_{2} \mathrm{O}(2: 1)$ ] to give 34a $(550 \mathrm{mg}, 60 \%)$ as a colorless oil and $\mathbf{3 4 b}(164 \mathrm{mg}, 18 \%)$ as a pale yellow oil.

34a: $[\alpha]_{\mathrm{D}}^{25}-40.1^{\circ}\left(c=0.86, \mathrm{CHCl}_{3}\right) .{ }^{1} \mathrm{H}-\mathrm{NMR}\left(\mathrm{CDCl}_{3}\right) \delta: 1.20(6 \mathrm{H}, \mathrm{s}$, $\left.\mathrm{CH}_{3}\right), 1.26\left(3 \mathrm{H}, \mathrm{t}, J=7.1 \mathrm{~Hz}, \mathrm{CO}_{2} \mathrm{CH}_{2} \mathrm{CH}_{3}\right), 1.50\left(9 \mathrm{H}, \mathrm{s}, \mathrm{C}\left(\mathrm{CH}_{3}\right)_{3}\right), 2.00(1 \mathrm{H}$, dd, $\left.J=13.7,0.9 \mathrm{~Hz}, 3-\mathrm{H}_{\beta}\right), 2.28\left(1 \mathrm{H}, \mathrm{dd}, J=13.7,10.3 \mathrm{~Hz}, 3-\mathrm{H}_{\alpha}\right), 2.42(3 \mathrm{H}$, $\left.\mathrm{s}, \mathrm{Ar}-\mathrm{CH}_{3}\right), 3.45(2 \mathrm{H}, \mathrm{s}, 5-\mathrm{H}), 4.09\left(2 \mathrm{H}, \mathrm{q}, J=7.1 \mathrm{~Hz}, \mathrm{CH}_{2} \mathrm{CH}_{3}\right), 4.20(1 \mathrm{H}, \mathrm{s}$, $\mathrm{OH}), 4.27(1 \mathrm{H}, \mathrm{dd}, J=10.3,0.9 \mathrm{~Hz}, 2-\mathrm{H}), 7.31(2 \mathrm{H}, \mathrm{d}, J=8.3 \mathrm{~Hz}, \mathrm{Ar}-\mathrm{H})$, $7.76(2 \mathrm{H}, \mathrm{d}, J=8.3 \mathrm{~Hz}, \mathrm{Ar}-\mathrm{H}) .{ }^{13} \mathrm{C}-\mathrm{NMR}\left(\mathrm{CDCl}_{3}\right) \delta: 14.03(\mathrm{q}), 21.44(\mathrm{q})$, $21.53(\mathrm{q}), 27.81(\mathrm{q}), 38.86(\mathrm{t}), 47.77(\mathrm{~s}), 56.98(\mathrm{t}), 60.21(\mathrm{~d}), 60.92(\mathrm{t}), 82.82$ (s), 83.10 (s), 127.62 (d), 129.69 (d), 135.03 (s), 143.80 (s), 172.63 (s), $175.82(\mathrm{~s})$. IR $v_{\max }^{\text {neat }} \mathrm{cm}^{-1}: 3442(\mathrm{OH}), 1720(\mathrm{C}=\mathrm{O})$. HR-MS (FAB) $m / z$ : 456.2055 (Calcd for $\mathrm{C}_{22} \mathrm{H}_{34} \mathrm{NO}_{7} \mathrm{~S}: 456.2056$ ).

34b: $[\alpha]_{\mathrm{D}}^{24}-43.6^{\circ}\left(c=0.82, \mathrm{CHCl}_{3}\right) .{ }^{1} \mathrm{H}-\mathrm{NMR}\left(\mathrm{CDCl}_{3}\right) \delta$ : $1.27(6 \mathrm{H}, \mathrm{s}$, $\left.\mathrm{CH}_{3}\right), 1.31\left(3 \mathrm{H}, \mathrm{t}, J=7.1 \mathrm{~Hz}, \mathrm{CH}_{2} \mathrm{C}_{3}\right), 1.50\left(9 \mathrm{H}, \mathrm{s}, \mathrm{C}\left(\mathrm{CH}_{3}\right)_{3}\right), 2.04(1 \mathrm{H}, \mathrm{m}$, $\left.3-\mathrm{H}_{\beta}\right), 2.14\left(1 \mathrm{H}\right.$, ddd, $\left.J=12.8,6.9,1.8 \mathrm{~Hz}, 3-\mathrm{H}_{\alpha}\right), 2.42\left(3 \mathrm{H}, \mathrm{s}, \mathrm{Ar}-\mathrm{CH}_{3}\right), 2.96$ $(1 \mathrm{H}, \mathrm{s}, \mathrm{OH}), 3.40\left(1 \mathrm{H}, \mathrm{dd}, J=11.7,1.8 \mathrm{~Hz}, 5-\mathrm{H}_{\alpha}\right), 3.62(1 \mathrm{H}, \mathrm{d}, J=11.7 \mathrm{~Hz}$, $\left.5-\mathrm{H}_{\beta}\right), 4.13\left(2 \mathrm{H}, \mathrm{q}, J=7.1 \mathrm{~Hz}, \mathrm{CH}_{2} \mathrm{CH}_{3}\right), 4.18(1 \mathrm{H}, \mathrm{dd}, J=9.9,6.9 \mathrm{~Hz}, 2-\mathrm{H})$,
$7.31(2 \mathrm{H}, \mathrm{d}, J=8.2 \mathrm{~Hz}, \mathrm{Ar}-\mathrm{H}), 7.79(2 \mathrm{H}, \mathrm{d}, J=8.2 \mathrm{~Hz}, \mathrm{Ar}-\mathrm{H}) .{ }^{13} \mathrm{C}-\mathrm{NMR}$ $\left(\mathrm{CDCl}_{3}\right) \delta: 14.04(\mathrm{q}), 21.76(\mathrm{q}), 21.90(\mathrm{q}), 27.78(\mathrm{q}), 31.60(\mathrm{~s}), 38.90(\mathrm{t})$, $47.19(\mathrm{~s}), 56.93(\mathrm{t}), 61.04(\mathrm{~d}), 61.37(\mathrm{t}), 81.82(\mathrm{~s}), 82.47(\mathrm{~s}), 127.91(\mathrm{~d})$ 129.52 (d), 134.64 (s), 143.59 (s), 171.18 (s), 177.12 (s). IR $v_{\max }^{\text {neat }} \mathrm{cm}^{-1}$ : $3506(\mathrm{OH}), 1736,1728(\mathrm{C}=\mathrm{O})$. HR-MS (FAB) $\mathrm{m} / \mathrm{z}$ : 456.2054 (Calcd for $\left.\mathrm{C}_{22} \mathrm{H}_{34} \mathrm{NO}_{7} \mathrm{~S}: 456.2056\right)$.

Aromatization of Compound 32 Giving 29 Thionyl chloride $(1.0 \mathrm{ml}$, $14 \mathrm{mmol}$ ) was added dropwise to a solution of compound 32a (129 mg, $0.30 \mathrm{mmol})$ in pyridine $(3.0 \mathrm{ml})$ at $0{ }^{\circ} \mathrm{C}$ and the whole was stirred at room temperature for $2 \mathrm{~h}$. The reaction mixture was concentrated under reduced pressure. Toluene $(12 \mathrm{ml})$ was added and the solution was concentrated under reduced pressure. This operation was repeated once. Water $(20 \mathrm{ml})$ was added to the residue and the whole was extracted with benzene $(20 \mathrm{ml} \times 2)$. The organic layer was washed with brine $(20 \mathrm{ml})$, dried over anhydrous $\mathrm{Na}_{2} \mathrm{SO}_{4}$, and concentrated under reduced pressure. The residue was dissolved in toluene $(2 \mathrm{ml})$ and DBU $(0.30 \mathrm{ml}, 2.0 \mathrm{mmol})$, and the solution was refluxed for $3 \mathrm{~h}$. The mixture was concentrated under reduced pressure and the residue was subjected to column chromatography on silica gel [hexane-AcOEt (1:1)] to give $\mathbf{2 9}(65 \mathrm{mg}, \mathbf{8 5 \%})$ as a colorless oil.

tert-Butyl 4-[1-(Ethoxycarbonyl)ethyl]pyrrole-2-carboxylate (35) Compound $35(72 \mathrm{mg}, 89 \%)$ was obtained as a pale yellow oil from 33a $(133 \mathrm{mg}, 0.30 \mathrm{mmol})$ in a manner similar to that described for $\mathbf{2 9}$ from $\mathbf{3 2}$ but using column chromatography on silica gel [benzene-Et $\left.{ }_{2} \mathrm{O}(5: 1)\right] .{ }^{1} \mathrm{H}$ $\operatorname{NMR}\left(\mathrm{CDCl}_{3}\right) \delta: 1.24\left(3 \mathrm{H}, \mathrm{t}, J=7.1 \mathrm{~Hz}, \mathrm{CH}_{2} \mathrm{CH}_{3}\right), 1.46(3 \mathrm{H}, \mathrm{d}, J=7.1 \mathrm{~Hz}$ benzylic $\left.\mathrm{CH}_{3}\right), 1.56\left(9 \mathrm{H}, \mathrm{s}, \mathrm{C}\left(\mathrm{CH}_{3}\right)_{3}\right), 3.64(1 \mathrm{H}, \mathrm{q}, J=7.1 \mathrm{~Hz}$, benzylic $\mathrm{H})$, $4.13\left(2 \mathrm{H}, \mathrm{q}, J=7.1 \mathrm{~Hz}, \mathrm{CH}_{2} \mathrm{CH}_{3}\right), 6.78(1 \mathrm{H}, \mathrm{s}, 3-\mathrm{H}), 6.83(1 \mathrm{H}, \mathrm{s}, 5-\mathrm{H}), 9.77$ $\left(1 \mathrm{H}\right.$, br s, NH). ${ }^{13} \mathrm{C}-\mathrm{NMR}\left(\mathrm{CDCl}_{3}\right) \delta: 14.16(\mathrm{q}), 18.44$ (q), 28.38 (q), 38.07 (d), 60.66 (t), 80.87 (s), 113.45 (d), 120.19 (d), 124.11 (s), 124.69 (s), 128.33 (d), 161.03 (s), 175.02 (s). IR $v_{\max }^{\text {neat }} \mathrm{cm}^{-1}: 3112(\mathrm{NH}), 1736,1676$ $(\mathrm{C}=\mathrm{O})$. HR-MS $m / z$ : 267.1469 (Calcd for $\mathrm{C}_{14} \mathrm{H}_{21} \mathrm{NO}_{4} \mathrm{~S}: 267.1471$ ).

tert-Butyl 4-[2-(Ethoxycarbonyl)propan-2-yl]pyrrole-2-carboxylate (36) Compound 36 (76 mg, 90\%) was obtained as a white solid from 34a $(137 \mathrm{mg}, 0.30 \mathrm{mmol})$ in a manner similar to that described for $\mathbf{2 9}$ from $\mathbf{3 2}$, but using column chromatography on silica gel [hexane-AcOEt $(10: 1)$ ]. It was recrystallized from hexane to give colorless prisms, mp $88-89^{\circ} \mathrm{C}{ }^{1} \mathrm{H}$ NMR $\left(\mathrm{CDCl}_{3}\right) \delta: 1.22\left(3 \mathrm{H}, \mathrm{t}, J=7.1 \mathrm{~Hz}, \mathrm{CH}_{2} \mathrm{CH}_{3}\right), 1.52(6 \mathrm{H}, \mathrm{s}$, benzylic $\left.\mathrm{CH}_{3}\right), 1.56\left(9 \mathrm{H}, \mathrm{s}, \mathrm{C}\left(\mathrm{CH}_{3}\right)_{3}\right), 4.12\left(2 \mathrm{H}, \mathrm{q}, J=7.1 \mathrm{~Hz}, \mathrm{CH}_{2} \mathrm{CH}_{3}\right), 6.80(1 \mathrm{H}, \mathrm{s}$, $3-\mathrm{H}), 6.83(1 \mathrm{H}, \mathrm{s}, 5-\mathrm{H}), 9.43(1 \mathrm{H}, \mathrm{s}, \mathrm{NH}) .{ }^{13} \mathrm{C}-\mathrm{NMR}\left(\mathrm{CDCl}_{3}\right) \delta: 14.09$ (q), 26.65 (q), 28.39 (q), 41.54 (s), 60.75 (t), 80.85 (s), 112.71 (d), 119.28 (d), $123.98(\mathrm{~s}), 130.38(\mathrm{~s}), 160.88(\mathrm{~s}), 176.70(\mathrm{~s}) . \mathrm{IR} v_{\max }^{\mathrm{KBr}} \mathrm{cm}^{-1}: 3311(\mathrm{NH})$, 1738, $1678(\mathrm{C}=\mathrm{O})$. MS $m / z: 281\left(\mathrm{M}^{+}\right)$. Anal. Calcd for $\mathrm{C}_{15} \mathrm{H}_{23} \mathrm{NO}_{4}: \mathrm{C}$, 64.03; H, 8.24; N, 4.98. Found: C, 64.19; H, 8.08; N, 4.68.

Acknowledgement This work was supported in part by the Special Research Fund of Hokuriku University.

\section{References}

1) Arakawa Y., Ohnishi M., Yoshimura N., Yoshifuji S., Chem. Pharm. Bull., 51, 1015-1020 (2003).

2) Portoghese P. S., Mikhail A. A., J. Org. Chem., 31, 1059-1062 (1966).

3) Tehrani K. A., Borremans D., Kimpe N. D., Tetrahedron, 55, 4133 4152 (1999).

4) Zimmer R., Collas M., Roth M., Reißig H.-U., Liebigs Ann. Chem., 1992, 709-714 (1992).

5) Walizei G. H., Breitmaier E., Synthesis, 1989, 337-340 (1989).

6) Barton D. H. R., Zard S., J. Chem. Soc., Chem. Commun., 16, $1098-$ 1100 (1985).

7) Robertson A. V., Francis J. E., Witkop B., J. Am. Chem. Soc., 84, 1709-1715 (1962).

8) Lash T. D., Hoehner M. C., J. Heterocycl. Chem., 28, 1671-1676 (1991).

9) Knight D. W., Redfern A. L., Gilmore J., J. Chem. Soc., Perkin Trans. 1, 2002, 622-628 (2002).

10) Cohnen E., Dewald R., Synthesis, 1987, 566-568 (1987).

11) Walizei G. H., Breitmaire E., Synthesis, 1989, 337-340 (1989).

12) Barton D. H. R., Kervagoret J., Zard S., Z., Tetrahedron, 46, 75877598 (1990).

13) Andreatta R. H., Nair V., Robertson A. V., Simpson W. R. J., Aust. J. Chem., 20, 1493-1509 (1967).

14) Barraclough P., Hudhomme P., Spray C. A., Young D. W., Tetrahedron, 51, 4195-4212 (1995).

15) Marcotte F.-A., Lubell W. D., Org. Lett., 4, $2601-2603$ (2002).

16) Imamoto T., Takiyama N., Nakamura K., Tetrahedron Lett., 26, 
$4763-4766$ (1985).

17) Imamoto T., Takiyama N., Nakamura K., Hatajima T., Kamiya Y., $J$. Am. Chem. Soc., 111, 4392- 4398 (1989).

18) Dormoy J.-R., Synthesis, 1982, 753-756 (1982).

19) Seyferth D., Heeren J. K., Grim S. O., J. Org. Chem., 26, 4783-4784 (1961).

20) Cheng M., De B., Almstead N. G., Pikul S., Dowty M. E., Dietsch C. R., Dunaway C. M., Gu F., Hsieh L. C., Janusz M. J., Taiwo Y. O., Natchus M. G., J. Med. Chem., 42, 5426-5436 (1999).

21) Pine S. H., Pettit R. J., Geib G. D., Cruz S. G., Gallego C. H., Tijerina
T., Pine R. D., J. Org. Chem., 50, 1212-1216 (1985).

22) Dinizo S. E., Freerksen R. W., Pabst W. E., Watt D. S., J. Org. Chem., 41, 2846-2849 (1976)

23) Rathke M. W., Lindert A., J. Org. Chem., 35, 3966-3967 (1970).

24) Santaniello E., Manzocchi A., Synthesis, 1977, 698-699 (1977).

25) Rapoport H., Bordner J., J. Org. Chem., 29, 2727-2731 (1961).

26) Smith J. A., Ng S., White J., Org. Biomol. Chem., 4, 2477-2482 (2006).

27) Demopoulos B. J., Anderson H. J., Loader C. E., Faber K., Can. J. Chem., 61, 2415-2422 (1983). 